\title{
A macroscale mixture theory analysis of deposition and sublimation rates during heat and mass transfer in dry snow
}

\author{
A. C. Hansen and W. E. Foslien \\ Department of Mechanical Engineering, University of Wyoming, Laramie, WY 82071, USA \\ Correspondence to: A. C. Hansen (hansen@uwyo.edu)
}

Received: 5 February 2015 - Published in The Cryosphere Discuss.: 5 March 2015

Revised: 11 August 2015 - Accepted: 21 August 2015 - Published: 23 September 2015

\begin{abstract}
The microstructure of a dry alpine snowpack is a dynamic environment where microstructural evolution is driven by seasonal density profiles and weather conditions. Notably, temperature gradients on the order of $10-20 \mathrm{~K} \mathrm{~m}^{-1}$, or larger, are known to produce a faceted snow microstructure exhibiting little strength. However, while strong temperature gradients are widely accepted as the primary driver for kinetic growth, they do not fully account for the range of experimental observations. An additional factor influencing snow metamorphism is believed to be the rate of mass transfer at the macroscale.

We develop a mixture theory capable of predicting macroscale deposition and/or sublimation in a snow cover under temperature gradient conditions. Temperature gradients and mass exchange are tracked over periods ranging from 1 to 10 days. Interesting heat and mass transfer behavior is observed near the ground, near the surface, as well as immediately above and below dense ice crusts. Information about deposition (condensation) and sublimation rates may help explain snow metamorphism phenomena that cannot be accounted for by temperature gradients alone.

The macroscale heat and mass transfer analysis requires accurate representations of the effective thermal conductivity and the effective mass diffusion coefficient for snow. We develop analytical models for these parameters based on first principles at the microscale. The expressions derived contain no empirical adjustments, and further, provide self consistent values for effective thermal conductivity and the effective diffusion coefficient for the limiting cases of air and solid ice. The predicted values for these macroscale material parameters are also in excellent agreement with numerical results based on microscale finite element analyses of representative volume elements generated from X-ray tomography.
\end{abstract}

\section{Introduction}

The thermodynamically active nature of snow, coupled with unusual high porosities, poses significant challenges to modeling heat and mass transfer in a snow cover. A primary driver in much of the research on this subject has been efforts to explain the evolving microstructure of snow that often occurs in a matter of hours or days. Notably, snow metamorphism, induced by strong temperature gradients in a snow cover, is known to produce a highly faceted microstructure, the presence of which results in extremely weak layers in a snow cover. Weak layers have been observed near the ground, near the surface, as well as above and below dense layers (e.g., ice crusts) within a snow cover.

While strong temperature gradients are widely accepted as the primary driver in temperature gradient metamorphism (TGM), they do not fully account for the range of experimental observations. For instance, slightly faceted crystal growth has been observed at low temperature gradient $\left(3 \mathrm{~K} \mathrm{~m}^{-1}\right)$ where rounded grains from sintering have normally been observed (Flin and Brzoska, 2008). In contrast, Pinzer and Schneebeli (2009) note that rounded grain forms have been observed in surface layers subjected to alternating temperature gradients of opposite direction.

An additional factor influencing snow metamorphism is believed to be the rate of mass transfer at the macroscale. The influence of mass transfer at the macroscale is often neglected for the simple fact that deposition (condensation) and sublimation rates caused by vapor diffusion and phase changes are not known in a typical macroscale analysis. Vapor diffusion and the associated phase changes at the macroscale pose modeling challenges in that it forces the macroscopic analysis toward a mixture theory where the ice and humid air constituents retain their identity. Mixture the- 
ory itself is a subject that has yet to fully mature and many open questions remain.

Implementing a macroscopic continuum mixture theory to elucidate the coupled heat and mass transfer phenomena occurring in snow is the central focus of this paper. We study the effects of mass transfer near the ground, near the surface including diurnal temperature effects, as well as adjacent to an ice crust within the snow cover. Heat and mass transfer rates are tracked over several different time periods ranging up to 10 days.

The mixture theory analysis developed herein requires an accurate assessment of macroscopic properties for effective thermal conductivity and the effective mass diffusion coefficient for snow. Determining these parameters requires an analysis of heat and mass transfer at the microscale. A major challenge in microstructural studies of snow metamorphism is the extremely complex 3-D structure of the ice phase.

Historically, generating an accurate geometric representation of the microstructure of snow and further connecting it to a subsequent heat and mass transfer analysis was simply not possible. However, in the last 2 decades, the use of X-ray computed tomography has profoundly altered experimental and theoretical research for snow at the microstructural level. Not only can one accurately capture the true 3-D snow microstructure, the evolution of the microstructure may be monitored in real time as metamorphism occurs. Furthermore, finite element analysis may be coupled to experimentally produced 3-D microstructures to model heat and mass transfer at the local scale.

High-fidelity microscale numerical models, coupled with $\mathrm{X}$-ray computer tomography, have been utilized by Riche and Schneebeli (2013) and Calonne et al. (2011) for predictions of macroscale effective thermal conductivity. Pinzer et al. (2012) and Flin and Brzoska (2008) used finite element analysis with X-ray tomography to address vapor diffusion. Evolution of the snow microstructure and determining an effective diffusion coefficient for snow are among their notable contributions.

Finite element predictions based on computer-generated $\mathrm{X}$-ray tomography snow structures provide an excellent foundation for determining material properties for effective thermal conductivity and the effective diffusion coefficient for snow. However, instead of utilizing finite element micromechanics to generate macroscale material properties, we rely on an interesting mathematical model developed by Foslien (1994). The analytical model produces results for effective thermal conductivity and the effective diffusion coefficient for snow that are in remarkable agreement with the finite element predictions cited above. The model also accounts for effective thermal conductivity and effective diffusion coefficient properties over the entire range of densities and temperatures possible for snow. The strong correlation of the analytical model material properties compared with results from microscale finite element analyses of snow lends confidence to using material parameters based on the analytical model in the macroscopic mixture theory analysis developed herein.

\section{Reflections on geometric scales: microscale vs. macroscale variables}

The critical heat and mass transfer mechanisms for snow metamorphism play out at two distinctly different geometric and time scales. At the microscale (on the order of millimeters) snow exhibits an extremely complex and evolving microstructure consisting of ice grains and humid air. At the macroscale, the geometric scale of interest is associated with the depth of the snow cover - typically on the order of meters. Macroscopic variables of interest include density, temperature, temperature gradient, as well as the mass flux of water vapor and the resulting deposition and sublimation that will occur within a snow cover. These macroscopic variables are fundamental drivers for snow structure evolution occurring at the microscale, thereby coupling local phenomena driving snow metamorphism with macroscale heat and mass transfer.

When developing a theory that transcends multiple geometric scales, attention must be paid to the transition from the microscale to the macroscale, commonly referred to as homogenization. An implicit requirement necessary for homogenization in an upscale process is appropriate separation of scales, both from a geometric and physical viewpoint. Auriault et al. (2009) provide extensive discussion of necessary conditions required for separation of scales, all of which are satisfied for the present work.

A notable aspect of the present homogenization process is that a mixture theory is introduced by defining snow at the macroscale to be a mixture composed of an ice constituent and a humid air constituent. The constituent variables may, in turn, be appropriately averaged to obtain the macroscale snow field variables. Allowing the constituents to retain their identity provides a vehicle to study mass transfer due to condensation and sublimation at the macroscale.

As a means of formalizing an upscaling process for snow, the concept of a representative volume element (RVE) is introduced. The RVE must be of sufficient size such that volume averages of the constituent variables do not change as the volume is increased.

Given an RVE, let $\phi_{\alpha}$ denote the volume fraction of constituent $\alpha$. The mixture constituents are immiscible, and the constituent volume fractions are space filling, leading to the relation

$\phi_{\mathrm{i}}+\phi_{\mathrm{ha}}=1$,

where subscripts (i) and (ha) denote the ice and humid air constituents, respectively.

The density of snow, $\rho$, is defined by the volume average of the local (microscale) density field, $\gamma_{\mathrm{m}}(\boldsymbol{x})$, that varies throughout the RVE, i.e., 
$\rho=\frac{1}{V} \int_{V} \gamma_{\mathrm{m}}(\boldsymbol{x}) \mathrm{d} V$,

where, for clarity, the local density may be expressed as

$\gamma_{\mathrm{m}}(\boldsymbol{x})=\gamma_{\mathrm{i}} \chi_{\mathrm{i}}(\boldsymbol{x})+\gamma_{\mathrm{ha}}\left(1-\chi_{\mathrm{i}}(\boldsymbol{x})\right)$

in terms of the indicator function $\chi_{\mathrm{i}}(\boldsymbol{x})$ of the ice phase. The subscript $(\mathrm{m})$ on the local density field is used to emphasize that the variable is defined at the microscale.

In the case of a mixture, the integral of Eq. (2) may be broken into an ice domain and a humid air domain as

$\rho=\frac{1}{V} \int_{V_{\mathrm{i}}} \gamma_{\mathrm{m}}(\boldsymbol{x}) \mathrm{d} V+\frac{1}{V} \int_{V_{\text {ha }}} \gamma_{\mathrm{m}}(\boldsymbol{x}) \mathrm{d} V$.

Moreover, the following macroscale constituent densities are introduced as

$\gamma_{\mathrm{i}}=\frac{1}{V_{\mathrm{i}}} \int_{V_{\mathrm{i}}} \gamma_{\mathrm{m}}(\boldsymbol{x}) \mathrm{d} V$,

and

$\gamma_{\text {ha }}=\frac{1}{V_{\text {ha }}} \int_{V_{\text {ha }}} \gamma_{\mathrm{m}}(\boldsymbol{x}) \mathrm{d} V$.

Noting Eqs. (4)-(6) leads to a volume average expression for the density of snow given by

$\rho=\phi_{\mathrm{i}} \gamma_{\mathrm{i}}+\phi_{\text {ha }} \gamma_{\text {ha }}$.

We emphasize that the mixture formulation is defined entirely at the macroscale. Hence, all variables in Eq. (7) represent macroscale quantities.

Following Özdemir et al. (2008), heat transfer properties are introduced into the micro-macro upscaling process by defining the macroscopic heat capacity as

$\left(\rho C^{\mathrm{V}}\right)=\frac{1}{V} \int_{V} \gamma_{\mathrm{m}}\left(C^{\mathrm{V}}\right)_{\mathrm{m}} \mathrm{d} V$,

where $C^{\mathrm{V}}$ is the specific heat at constant volume. This equation provides a definition for the specific heat of snow yielding consistent values of heat capacity at both scales. Following the same development as for the density of snow leads to the relation

$\left(\rho C^{\mathrm{V}}\right)=\phi_{\mathrm{i}}\left(\gamma_{\mathrm{i}} C_{\mathrm{i}}^{\mathrm{V}}\right)+\phi_{\mathrm{ha}}\left(\gamma_{\mathrm{ha}} C_{\mathrm{ha}}^{\mathrm{V}}\right)$,

where the heat capacity for constituent $\alpha$ is given by

$\left(\gamma_{\alpha} C_{\alpha}^{\mathrm{V}}\right)=\frac{1}{V_{\alpha}} \int_{V_{\alpha}} \gamma_{\mathrm{m}}\left(C^{\mathrm{V}}\right)_{\mathrm{m}} \mathrm{d} V$.

Özdemir et al. (2008) further enforces consistency of the stored heat at the microscale and macroscale through the relation $\left(\rho C^{\mathrm{V}}\right) \theta=\frac{1}{V} \int_{\mathrm{V}} \gamma_{\mathrm{m}}\left(C^{\mathrm{V}}\right)_{\mathrm{m}} \theta_{\mathrm{m}} \mathrm{d} V$

where $\theta_{\mathrm{m}}$ and $\theta$ represent the local temperature and macroscale temperature, respectively. Again, the integral of Eq. (11) may be separated into an ice constituent and a humid air constituent as

$$
\begin{aligned}
\left(\rho C^{\mathrm{V}}\right) & =\phi_{\mathrm{i}}\left(\frac{1}{V_{\mathrm{i}}} \int_{V_{\mathrm{i}}} \gamma_{\mathrm{m}}\left(C^{\mathrm{V}}\right)_{\mathrm{m}} \theta_{\mathrm{m}} \mathrm{d} V\right) \\
& +\phi_{\mathrm{ha}}\left(\frac{1}{V_{\text {ha }}} \int_{V_{\text {ha }}} \gamma_{\mathrm{m}}\left(C^{\mathrm{V}}\right)_{\mathrm{m}} \theta_{\mathrm{m}} \mathrm{d} V\right) .
\end{aligned}
$$

Constituent temperatures, $\theta_{\mathrm{i}}$ and $\theta_{\mathrm{ha}}$, are introduced through the relations

$\gamma_{\mathrm{i}} C_{\mathrm{i}}^{\mathrm{V}} \theta_{\mathrm{i}}=\frac{1}{V_{\mathrm{i}}} \int_{V_{\mathrm{i}}} \gamma_{\mathrm{m}}\left(C^{\mathrm{V}}\right)_{\mathrm{m}} \theta_{\mathrm{m}} \mathrm{d} V$,

and

$\gamma_{\text {ha }} C_{\text {ha }}^{\mathrm{V}} \theta_{\text {ha }}=\frac{1}{V_{\text {ha }}} \int_{V_{\text {ha }}} \gamma_{\mathrm{m}}\left(C^{\mathrm{V}}\right)_{\mathrm{m}} \theta_{\mathrm{m}} \mathrm{d} V$.

The heat capacity is heterogeneous at the microscale but homogeneous in the ice phase, leading to a volume average temperature for ice given by

$\theta_{\mathrm{i}}=\frac{1}{V_{\mathrm{i}}} \int_{V_{\mathrm{i}}} \theta_{\mathrm{m}} \mathrm{d} V$

For the range of temperatures of interest, the mass fraction of water vapor in dry air is on the order of $10^{-3}$. Hence, the thermal properties of the humid air may be taken to be those of dry air, and the heat capacity of dry air is constant for the temperature variations seen at the microscale. This condition leads to a volume average definition for the temperature of the humid air constituent given by

$\theta_{\text {ha }}=\frac{1}{V_{\text {ha }}} \int_{V_{\text {ha }}} \theta_{\mathrm{m}} \mathrm{d} V$.

The temperature of snow may be determined from

$\left(\rho C^{\mathrm{V}}\right) \theta=\phi_{\mathrm{i}}\left(\gamma_{\mathrm{i}} C_{\mathrm{i}}^{\mathrm{V}}\right) \theta_{\mathrm{i}}+\phi_{\mathrm{ha}}\left(\gamma_{\mathrm{ha}} C_{\mathrm{ha}}^{\mathrm{V}}\right) \theta_{\mathrm{ha}}$.

Hence, the temperature of snow does not follow the constituent volume averaging found for the heat capacity (Eq. 9) and the density (Eq. 4) but rather is based on a volume average weighted by the constituent heat capacities.

The temperature gradient at the microscale is a critical parameter driving temperature gradient metamorphism. To this end, volume averaged temperature gradients for the ice and humid air constituents are introduced as 
- $\nabla \theta_{\mathrm{i}}$ ice temperature gradient

- $\nabla \theta_{\text {ha }}$ humid air temperature gradient

where, for example,

$\nabla \theta_{\mathrm{i}}=\frac{1}{V_{\mathrm{i}}} \int_{V_{\mathrm{i}}} \nabla_{x} \theta_{\mathrm{m}}(\boldsymbol{x}) \mathrm{d} V$.

The subscript $x$ on the gradient operator in Eq. (18) is used to emphasize that the gradient applies at the microscale.

Given appropriate boundary conditions for the RVE, the macroscale temperature gradient for snow satisfies the volume weighted averaging:

$\nabla \theta=\phi_{\mathrm{i}} \nabla \theta_{\mathrm{i}}+\phi_{\mathrm{ha}} \nabla \theta_{\text {ha }}$.

Özdemir et al. (2008) develop the specific boundary conditions for the RVE that are necessary to satisfy Eq. (19). These boundary conditions are precisely the ones used by Pinzer et al. (2012) and Riche and Schneebeli (2013) in their finite element analyses of heat and mass transfer at the microscale.

Finally, it is extremely important to recognize differences in behavior between local (microscale) temperature gradients and the volume averaged macroscale temperature gradient. For instance, Pinzer et al. (2012) provide a figure of the local temperature gradients in an RVE for an applied macroscale temperature gradient of $50 \mathrm{~K} \mathrm{~m}^{-1}$. The color bar for the microscale temperature gradient indicates that local values of the temperature gradient are as high as $300 \mathrm{~K} \mathrm{~m}^{-1}$. The high local values of the temperature gradient compared to the macroscopic temperature gradient must be kept in mind when interpreting macroscopic results, as it is the local temperature gradients that drive metamorphism. Hence, when macroscale temperature gradients are presented as computed by the mixture theory analysis, it is not unreasonable to assume the microscale temperature gradients may be an order of magnitude higher in some areas of the RVE.

\section{A mixture theory model for macroscale heat and mass transfer}

The common phase changes occurring in snow have motivated several studies using variants of mixture theories. Morland et al. (1990) and Bader and Weilenmann (1992) developed a four constituent mixture theory for snow where one of the constituents was water. Phenomena such as percolation, melting, and freezing are addressed, and momentum balance plays a significant role in the work. The present work does not involve momentum balance, nor does it allow for a water constituent.

Gray and Morland (1994) developed a mixture theory for dry snow based on constituents of ice and dry air. Their work is in sharp contrast to the present study where water vapor is a critical component of the development. Indeed, the emphasis of the present work is the prediction of deposition and/or sublimation of water vapor at the macroscale.

Adams and Brown (1990) studied heat and mass transfer in snow using a classical form of mixture theory where water vapor was included. Their work focused on non-equilibrium conditions of the constituents, whereas the present work is based on equilibrium of constituent temperatures and a saturated vapor density. Equilibrium vs. non-equilibrium conditions amounts to a focus on different time scales.

Aside from the different areas of emphasis in the study of phase change phenomena in snow, the mixture theories cited are based on a classical theory of mixtures, whereas the present work is largely based on a volume fraction mixture theory (Hansen et al., 1991). The volume fraction theory produces the same balance equations found in the classical developments of mixture theory. However, the summed constituent balance equations are not forced to reduce to those of a single continuum except for the special case of a nondiffusing mixture. As a result of relaxing this constraint, the physical definitions of mixture variables as well as the constraints on mass, momentum, and energy interaction terms assume more appealing forms. We rely on the physical arguments of Sect. 2 to define mixture quantities of interest.

Albert and McGilvary (1992) incorporated the effects of mass diffusion in a heat and mass transfer analysis of snow centered on forced convection caused by windy conditions close to the snow surface, a phenomenon known as wind pumping. The equations developed involve a velocity of the humid air and conditions where the snow is not assumed to be saturated with water vapor. These conditions only occur in snow under extreme circumstances.

Foslien (1994) performed a dimensional analysis of the conditions needed for convection and showed the Rayleigh number for typical snow conditions was 1-2 orders of magnitude below what is needed for the onset of convection. As a consequence, convection is not considered, and the present paper develops a theory with no air velocity, and further, a saturated vapor density.

The work of Calonne et al. (2014a) is perhaps the most closely related to the present work in that they developed the governing equations for macroscopic heat and water vapor transfer in dry snow by homogenization involving a multiscale expansion. We draw comparisons of their work for the governing macroscale equations as well as the expressions for effective thermal conductivity and the effective diffusion coefficient in snow.

A unique aspect of the present approach is that analytical models, grounded in first principles at the microscale, are developed for the effective thermal conductivity and the effective diffusion coefficient in snow. By starting at the microscale, albeit with idealized microstructures, we are afforded the advantage of using the true thermal conductivities of ice $\left(k_{\mathrm{i}}\right)$ and humid air $\left(k_{\mathrm{ha}}\right)$ as well as the known diffusion coefficient of water vapor in air $\left(D_{\mathrm{v}-\mathrm{a}}\right)$. The resulting 
models for the effective thermal conductivity of snow and the effective diffusion coefficient for snow contain no empirical adjustments and are in remarkable agreement with highfidelity numerical predictions of these parameters based on snow microstructures obtained from X-ray tomography. The models also generate an analytical description of the separation of heat transfer due to mass diffusion and heat transfer due to conduction.

Consistent with the discussion on homogenization, we consider snow at the macroscale to be a two-constituent mixture consisting of ice and humid air. The humid air itself is treated as a mixture of water vapor and air. A schematic of the mixture theory analysis is shown in Fig. 1.

For the temperatures and pressures encountered in snow, the humid air may be treated as a mixture of two ideal gases where each gas occupies the same volume, i.e.,

$\phi_{\mathrm{ha}}=\phi_{\mathrm{v}}=\phi_{\mathrm{a}}$,

where subscripts (v) and (a) represent water vapor and dry air, respectively. An important consequence of representing the humid air as a mixture of ideal gases is that both the water vapor and the air behave as though the other gas is not present, thereby greatly simplifying the analysis and allowing one to draw on classical results for ideal gases.

The balance equations for mass, momentum, and energy for a constituent, $\alpha$, are given by (Hansen, 1989; Hansen et al., 1991) as follows:

Mass balance

$\frac{\partial \rho_{\alpha}}{\partial t}+\nabla \cdot\left(\rho_{\alpha} \boldsymbol{v}_{\alpha}\right)=\hat{c}_{\alpha}$

Momentum balance

$\rho_{\alpha} \boldsymbol{a}_{\alpha}=\nabla \cdot \boldsymbol{T}_{\alpha}+\rho_{\alpha} \boldsymbol{g}+\hat{\boldsymbol{p}}_{\alpha}$,

Energy balance

$\rho_{\alpha} \dot{u}_{\alpha}=\operatorname{tr}\left(\boldsymbol{T}_{\alpha} \cdot \boldsymbol{L}_{\alpha}\right)+\rho_{\alpha} r_{\alpha}-\nabla \cdot \boldsymbol{q}_{\alpha}+\hat{e}_{\alpha}$.

In the above, $\boldsymbol{v}_{\alpha}$ and $\boldsymbol{a}_{\alpha}$ represent the velocity and acceleration of constituent $\alpha$, respectively, while $\boldsymbol{L}_{\alpha}$ represents the velocity gradient; $u_{\alpha}$ is the internal energy, $r_{\alpha}$ is the heat supply (notably radiation), and $\boldsymbol{g}$ is the gravity vector. The dispersed density of constituent $\alpha$ is denoted by $\rho_{\alpha}$ and is related to the true density, $\gamma_{\alpha}$, as

$\rho_{\alpha}=\phi_{\alpha} \gamma_{\alpha}$

Whereas the volume fraction, $\phi_{\alpha}$, appears explicitly in the definition of the dispersed density, $\rho_{\alpha}$, the partial stress, $\boldsymbol{T}_{\alpha}$, and the energy flux, $\boldsymbol{q}_{\alpha}$, are implicitly scaled by the volume fraction. Finally, $\hat{c}_{\alpha}, \hat{\boldsymbol{p}}_{\alpha}$, and $\hat{e}_{\alpha}$ represent mass, momentum, and energy supply terms that arise from interactions between constituents. Following Hansen et al. (1991), the mixture theory supply terms satisfy the appealing restrictions

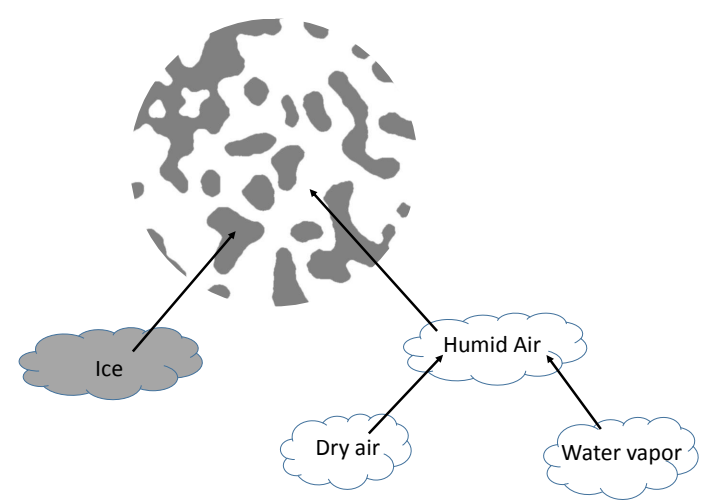

Figure 1. Schematic showing a continuum point of snow with the associated constituents for a mixture theory analysis.

$\sum_{\alpha} \hat{c}_{\alpha}=0$,

$\sum_{\alpha} \hat{\boldsymbol{p}}_{\alpha}=\mathbf{0}$,

and

$\sum_{\alpha} \hat{e}_{\alpha}=0$.

In what follows, the mixture theory balance equations are further specialized to study the macroscale coupled heat and mass transfer problem for snow.

\subsection{Ice constituent mass balance}

The balance of mass for the ice phase is given by

$\frac{\partial \rho_{\mathrm{i}}}{\partial t}+\nabla \cdot\left(\rho_{\mathrm{i}} \boldsymbol{v}_{\mathrm{i}}\right)=\hat{c}_{\mathrm{i}}$.

Assuming the mass supply is positive during condensation, we can write

$\hat{c}=\hat{c}_{\mathrm{i}}=-\hat{c}_{\mathrm{ha}}$.

Neglecting any settling velocity leads to a mass balance for the ice constituent given by

$\gamma_{\mathrm{i}} \frac{\partial \phi_{\mathrm{i}}}{\partial t}=\hat{c}$,

where the mass density of ice is taken as constant at $917 \mathrm{~kg} \mathrm{~m}^{-3}$.

\subsection{Water vapor mass balance}

The development of the humid air mass balance differs from that of the ice constituent in that we begin at the microscale. Furthermore, only the mass balance of the water vapor is considered because the air acts only as a medium through which the water vapor diffuses. 
Mass transfer of the water vapor may be expressed as (Bird and Lightfoot, 1960)

$\gamma_{\mathrm{v}} \boldsymbol{v}_{\mathrm{v}}=\frac{\gamma_{\mathrm{v}}}{\gamma_{\mathrm{ha}}}\left(\gamma_{\mathrm{a}} \boldsymbol{v}_{\mathrm{a}}+\gamma_{\mathrm{v}} \boldsymbol{v}_{\mathrm{v}}\right)+\boldsymbol{j}_{\mathrm{v}}$

Equation (31) says that the mass flux of the water vapor is due to the bulk fluid motion (the barycentric velocity) plus a relative velocity due to diffusion. In the absence of a pressure gradient, the barycentric velocity is zero, i.e.,

$\gamma_{\text {ha }} \boldsymbol{v}_{\text {ha }}=\left(\gamma_{\mathrm{a}} \boldsymbol{v}_{\mathrm{a}}+\gamma_{\mathrm{v}} \boldsymbol{v}_{\mathrm{v}}\right)=0$.

Mass balance due to diffusion may be expressed in the form of Fick's law (Bird and Lightfoot, 1960) as

$\boldsymbol{j}_{\mathrm{v}}=-\gamma_{\mathrm{ha}} D_{\mathrm{v}-\mathrm{a}} \nabla_{x}\left(\frac{\gamma_{\mathrm{v}}}{\gamma_{\mathrm{ha}}}\right)$,

where $D_{\mathrm{v}-\mathrm{a}}$ is the binary diffusion coefficient for water vapor in air and $\nabla_{x}$ denotes the gradient operator at the microscale.

The diffusive flux can be expanded to give

$\boldsymbol{j}_{\mathrm{v}}=-D_{\mathrm{v}-\mathrm{a}} \nabla_{x} \gamma_{\mathrm{v}}+\frac{\gamma_{\mathrm{v}}}{\gamma_{\mathrm{ha}}} D_{\mathrm{v}-\mathrm{a}} \nabla_{x} \gamma_{\mathrm{ha}}$,

but the second term on the right is negligibly small because the mass fraction of saturated water vapor in air at $273 \mathrm{~K}$ is about $4 \times 10^{-3}$. Hence, mass transfer of water vapor at the microscale may be described by

$\gamma_{\mathrm{v}} \boldsymbol{v}_{\mathrm{v}}=-D_{\mathrm{v}-\mathrm{a}} \nabla_{x} \gamma_{\mathrm{v}}$

In the transition to the macroscale, the same physical principles apply but one must now use an effective diffusion coefficient for water vapor. The need to introduce an effective diffusion coefficient for water vapor is attributed to the presence of the ice microstructure in snow. Specifically, the presence of the ice constituent introduces vapor transfer mechanisms that both enhance and retard mass transfer of water vapor when compared to a medium of humid air only. These mass transfer mechanisms are briefly discussed in Sect. 5.3.

Defining $D_{\mathrm{s}}^{\text {eff }}$ as the effective diffusion coefficient for the humid air constituent at the macroscale follows

$\phi_{\mathrm{v}} \gamma_{\mathrm{v}} \boldsymbol{v}_{\mathrm{v}}=\rho_{\mathrm{v}} \boldsymbol{v}_{\mathrm{v}}=-D_{\mathrm{s}}^{\mathrm{eff}} \nabla \gamma_{\mathrm{v}}$

where $\boldsymbol{v}_{\mathrm{v}}$ and $\gamma_{\mathrm{v}}$ now represent appropriately volume averaged macroscale variables. Note that the mass flux of water vapor is based on the dispersed density, $\rho_{\mathrm{v}}$, in order to account for the reduced volume occupied by the humid air in the mixture. Finally, since only the humid air constituent is associated with diffusion in a mixture of ice and humid air, $D_{\mathrm{s}}^{\text {eff }}$ also represents the effective diffusion coefficient for snow.

Again, noting air is simply the medium for mass transfer of water vapor, the balance of mass for the vapor phase may be written as $\frac{\partial \rho_{\mathrm{v}}}{\partial t}+\nabla \cdot\left(\rho_{\mathrm{v}} \boldsymbol{v}_{\mathrm{v}}\right)=\hat{c}_{\mathrm{v}}$

Substitution of the diffusive flux into Eq. (37) and noting $\hat{c}_{\mathrm{V}}=\hat{c}_{\text {ha }}=-\hat{c}$ leads to

$\frac{\partial \rho_{\mathrm{v}}}{\partial t}-\nabla \cdot\left(D_{\mathrm{s}}^{\mathrm{eff}} \nabla \gamma_{\mathrm{v}}\right)=-\hat{c}$.

Expanding the time derivative of the dispersed density of the water vapor gives

$\frac{\partial \rho_{\mathrm{v}}}{\partial t}=\gamma_{\mathrm{v}} \frac{\partial \phi_{\mathrm{v}}}{\partial t}+\phi_{\mathrm{v}} \frac{\partial \gamma_{\mathrm{v}}}{\partial t}$

but

$\frac{\partial \phi_{\mathrm{v}}}{\partial t}=\frac{\partial \phi_{\mathrm{ha}}}{\partial t}=-\frac{\partial \phi_{\mathrm{i}}}{\partial t}$.

The above results, along with the mass balance for the ice constituent (Eq. 30), can be used to write Eq. (38) as

$\phi_{\mathrm{v}} \frac{\partial \gamma_{\mathrm{v}}}{\partial t}-\nabla \cdot\left(D_{\mathrm{s}}^{\mathrm{eff}} \nabla \gamma_{\mathrm{v}}\right)=\hat{c}\left(\frac{\gamma_{\mathrm{v}}}{\gamma_{\mathrm{i}}}-1\right)$,

but the quantity $\frac{\gamma_{\mathrm{v}}}{\gamma_{\mathrm{i}}} \ll 1$. Neglecting this term and noting $\phi_{\mathrm{v}}=\phi_{\mathrm{ha}}$, the mass balance equation for the water vapor becomes

$\phi_{\mathrm{ha}} \frac{\partial \gamma_{\mathrm{v}}}{\partial t}-\nabla \cdot\left(D_{\mathrm{s}}^{\mathrm{eff}} \nabla \gamma_{\mathrm{v}}\right)=-\hat{c}$.

Equation (42) states that changes in the water vapor density at the macroscale are due to the divergence of the water vapor flux and sublimation or condensation as defined through the mass supply.

\subsection{Momentum balance}

The momentum balance for the ice phase can be used to find the stress and strain in the ice phase. However, the effect that the ice stress has on the vapor density of the water is neglected, so the ice phase momentum balance is not considered further.

The momentum balance for the humid air phase becomes important when bulk fluid motion occurs as in the case of convection. Foslien (1994) has shown the Rayleigh number for a typical snow cover is more than an order of magnitude below the critical value for the onset of convection, so convection is unlikely to occur except in extreme circumstances. Therefore, the momentum balance of the humid air phase is not considered further.

\subsection{Ice constituent energy balance}

The energy balance for the ice constituent may be expressed at the macroscale as

$\rho_{\mathrm{i}} \dot{u}_{\mathrm{i}}=\operatorname{tr}\left(\boldsymbol{T}_{\mathrm{i}} \cdot \boldsymbol{L}_{\mathrm{i}}\right)+\rho_{\mathrm{i}} r_{\mathrm{i}}-\nabla \cdot \boldsymbol{q}_{\mathrm{i}}+\hat{e}_{\mathrm{i}}$. 
In the above, any velocity gradient in the ice, $\boldsymbol{L}_{\mathrm{i}}$, is attributed to settling and may be neglected. Moreover, heat generation from solar radiation is also neglected but could easily be included as Colbeck (1989) and McComb et al. (1992) have done. These assumptions reduce the energy balance for ice to

$\rho_{\mathrm{i}} \dot{u}_{\mathrm{i}}=-\nabla \cdot \boldsymbol{q}_{\mathrm{i}}+\hat{e}_{\mathrm{i}}$.

The internal energy of the non-deforming ice is assumed to be a function of temperature only and is given by

$u_{\mathrm{i}}=C_{\mathrm{i}}^{\mathrm{V}}\left(\theta_{\mathrm{i}}-\theta_{\mathrm{ref}}\right)$,

where $C_{i}^{\mathrm{V}}$ is the specific heat of ice at constant volume and $\theta_{\text {ref }}$ is the reference temperature. The heat flux at the macroscale is expressed as Fourier's law of heat conduction as

$\boldsymbol{q}_{\mathrm{i}}=-\phi_{\mathrm{i}} \mathrm{k}_{\mathrm{i}}^{\mathrm{eff}} \nabla \theta_{\mathrm{i}}$,

where $k_{\mathrm{i}}^{\text {eff }}$ is the effective thermal conductivity for the ice phase in snow. This parameter should not be confused with the thermal conductivity of pure ice $\left(k_{\mathrm{i}}\right)$ as differences arise due to the complex microstructural network of the ice phase in snow. The tortuosity of the ice phase, for example, plays a role in $k_{\mathrm{i}}^{\text {eff }}$. The only microstructure where $k_{\mathrm{i}}$ and $k_{\mathrm{i}}^{\text {eff }}$ would be equal for 1-D heat transfer would be the pore microstructure discussed in the present paper. In a 3-D analysis of snow, the two parameters are fundamentally different.

Combining Eqs. (44)-(46), the energy balance for the ice phase is given by

$\phi_{\mathrm{i}} \gamma_{\mathrm{i}} C_{\mathrm{i}}^{\mathrm{V}} \frac{\partial \theta_{\mathrm{i}}}{\partial t}=\nabla \cdot\left(\phi_{\mathrm{i}} k_{\mathrm{i}}^{\text {eff }} \nabla \theta_{\mathrm{i}}\right)+\hat{e}_{\mathrm{i}}$.

\subsection{Humid air constituent energy balance}

As with the ice phase, the work term and the energy source term of the humid air constituent are neglected, thereby reducing the energy equation to

$\rho_{\mathrm{ha}} \dot{u}_{\mathrm{ha}}=-\nabla \cdot \boldsymbol{q}_{\mathrm{ha}}+\hat{e}_{\mathrm{ha}}$.

The internal energy for the humid air mixture of ideal gases is given by

$\gamma_{\text {ha }} u_{\mathrm{ha}}=\gamma_{\mathrm{a}} C_{\mathrm{a}}^{\mathrm{V}}\left(\theta_{\mathrm{ha}}-\theta_{\mathrm{ref}}\right)+\gamma_{\mathrm{v}}\left(C_{\mathrm{v}}^{\mathrm{V}}\left(\theta_{\mathrm{ha}}-\theta_{\mathrm{ref}}\right)+u_{\mathrm{sg}}\right)$,

where $u_{\mathrm{sg}}$ is the latent heat of sublimation of ice. The above assumes the reference value of the internal energy of ice was set to zero as was the case.

The definition for the energy flux vector for a mixture may be written as (Bird and Lightfoot, 1960)

$\boldsymbol{q}=\boldsymbol{q}^{\mathrm{c}}+\boldsymbol{q}^{\mathrm{d}}$,

where $\boldsymbol{q}^{\mathrm{c}}$ is the conductive flux and $\boldsymbol{q}^{\mathrm{d}}$ represents a "contribution from the interdiffusion of various species present". In the case of a mixture of water vapor and air, the energy flux is given by

$\boldsymbol{q}_{\mathrm{ha}}=-k_{\mathrm{ha}} \boldsymbol{\nabla}_{x} \theta_{\mathrm{ha}}+u_{\mathrm{sg}} \gamma_{\mathrm{v}} \boldsymbol{v}_{\mathrm{v}}$,

where $\gamma_{\mathrm{v}} \boldsymbol{v}_{\mathrm{v}}$, is the mass flux of water vapor diffusing through air.

Now consider snow at the macroscale composed of a mixture of humid air and ice. At this scale, Eq. (51) must be modified as

$\boldsymbol{q}_{\text {ha }}=-\phi_{\text {ha }} k_{\text {ha }}^{\text {eff }} \nabla \theta_{\text {ha }}+\phi_{\text {ha }} u_{\mathrm{sg}} \gamma_{\mathrm{v}} \boldsymbol{v}_{\mathrm{v}}$.

The interpretation of the volume fraction in each term on the right-hand side of the above equation is clear when one views the energy flux across a surface of a macroscale control volume of snow. Specifically, the true energy flux of humid air must be scaled by the area fraction of the humid air at the control surface. From quantitative stereology, the area fraction is equal to the volume fraction, resulting in Eq. (52).

Noting Eq. (36), mass transfer of the humid air may be expressed as a diffusive flux, leading to

$\boldsymbol{q}_{\mathrm{ha}}=-\phi_{\mathrm{ha}} k_{\mathrm{ha}}^{\mathrm{eff}} \nabla \theta_{\mathrm{ha}}-u_{\mathrm{sg}} D_{\mathrm{s}}^{\mathrm{eff}} \nabla \gamma_{\mathrm{v}}$,

where $D_{\mathrm{s}}^{\text {eff }}$ represents an effective diffusion coefficient for snow.

As in the case of the ice phase, one must recognize that $k_{\text {ha }}^{\text {eff }}$ represents an effective thermal conductivity of the humid air in snow, and this parameter is different from the true thermal conductivity of humid air as a pure substance. The difference in the two parameters is again attributed to the complex microstructure of the humid air phase in snow. In brief, just as the effective thermal conductivity of snow, $k_{\mathrm{s}}^{\text {eff }}$ is influenced by microstructure, so are $k_{\mathrm{i}}^{\text {eff }}$ and $k_{\mathrm{ha}}^{\text {eff }}$ as all three parameters are macroscale quantities. As such, they depend on a host of microstructural variables other than temperature.

Substituting Eqs. (49) and (53) into Eq. (48) leads to

$$
\begin{aligned}
\phi_{\mathrm{ha}} & \left(\gamma_{\mathrm{a}} C_{\mathrm{a}}^{\mathrm{V}}+\gamma_{\mathrm{v}} C_{\mathrm{v}}^{\mathrm{V}}\right) \frac{\partial \theta_{\mathrm{ha}}}{\partial t}+u_{\mathrm{sg}}\left(\phi_{\mathrm{ha}} \frac{\partial \gamma_{\mathrm{v}}}{\partial t}-\nabla \cdot\left(D_{\mathrm{s}}^{\mathrm{eff}} \nabla \gamma_{\mathrm{v}}\right)\right) \\
& =\nabla \cdot\left(\phi_{\mathrm{ha}} k_{\mathrm{ha}}^{\mathrm{eff}} \nabla \theta_{\mathrm{ha}}\right)+\hat{e}_{\mathrm{ha}},
\end{aligned}
$$

but

$\hat{c}=\nabla \cdot\left(D_{\mathrm{s}}^{\mathrm{eff}} \nabla \gamma_{\mathrm{v}}\right)-\phi_{\mathrm{ha}} \frac{\partial \gamma_{\mathrm{v}}}{\partial t}$,

from the mass balance of the water vapor given by Eq. (42). Therefore, Eq. (54), governing the energy balance of humid air, assumes the form

$\phi_{\text {ha }}\left(\gamma_{\mathrm{a}} C_{\mathrm{a}}^{\mathrm{V}}+\gamma_{\mathrm{v}} C_{\mathrm{v}}^{\mathrm{V}}\right) \frac{\partial \theta_{\mathrm{ha}}}{\partial t}=\nabla \cdot\left(\phi_{\mathrm{ha}} \mathrm{ha}_{\mathrm{ha}}^{\mathrm{eff}} \nabla \theta_{\mathrm{ha}}\right)+\hat{e}_{\mathrm{ha}}+u_{\mathrm{sg}} \hat{c}$.

Hence, the change in internal energy for the humid air is attributed to the divergence of the heat flux, energy exchange with the ice constituent through the energy supply, and energy exchange through phase changes accounted for by the mass supply. 


\section{Separation of scales: macroscale observations}

In this section, we discuss some observations that lead to important simplifications in the macroscale heat and mass transfer solution. Moreover, we demonstrate separation of the time scales for local and global heat and mass transfer, a condition required for homogenization.

\subsection{Macroscale temperatures}

An important simplification in the analysis of heat and mass transfer at the macroscale is to assume the constituent temperatures are equal and write

$\theta=\theta_{\mathrm{i}}=\theta_{\mathrm{ha}}$,

where $\theta$ is the macroscale temperature of snow. Justification for assuming the ice and humid air temperatures are equal starts by writing a 1-D heat conduction equation at the microscale given by

$$
\frac{\partial \theta_{\alpha}}{\partial t}=\left(\frac{k_{\alpha}}{\gamma_{\alpha} C_{\alpha}^{\mathrm{V}}}\right) \frac{\partial^{2} \theta_{\alpha}}{\partial x^{2}}
$$

Equation (57) is non-dimensionalized by introducing the following dimensionless variables:

$t^{*}=t / t_{\mathrm{o}}, x^{*}=x / L_{\mathrm{c}}$, and $\theta^{*}=\frac{\theta-\theta_{\text {init }}}{\theta_{\mathrm{f}}-\theta_{\text {init }}}$.

The resulting non-dimensional equation is

$\frac{\partial \theta^{*}}{\partial t^{*}}=\left(\frac{t_{\mathrm{o}} k_{\alpha}}{L_{\mathrm{c}}^{2} \gamma_{\alpha} C_{\alpha}^{\mathrm{V}}}\right) \frac{\partial^{2} \theta^{*}}{\partial x^{* 2}}$.

The time scale, $t_{\mathrm{o}}^{\text {micro }}$, for heat conduction on the microscale is introduced as

$t_{\mathrm{o}}^{\mathrm{micro}}=\frac{\gamma_{\alpha} C_{\alpha}^{\mathrm{V}} L_{\mathrm{c}}^{2}}{k_{\alpha}}$.

The time scale, $t_{\mathrm{o}}^{\text {macro }}$, for heat conduction in a snow cover is similarly defined as

$t_{\mathrm{o}}^{\text {macro }}=\frac{\left(\phi_{\mathrm{i}} \gamma_{\mathrm{i}} C_{\mathrm{i}}^{\mathrm{V}}+\phi_{\mathrm{ha}} \gamma_{\mathrm{ha}} C_{\mathrm{ha}}^{\mathrm{V}}\right) H^{2}}{k_{\mathrm{s}}^{\text {eff }}}$,

where $H$ is the height of the snowpack and $k_{\mathrm{s}}^{\mathrm{eff}}$ represents the effective thermal conductivity for snow.

Riche and Schneebeli (2013) provide an expression for the effective thermal conductivity of snow as a function of snow density. Assuming a snow density of $200 \mathrm{~kg} \mathrm{~m}^{-3}$, a depth of $1 \mathrm{~m}$, and a microscale characteristic length of $1 \mathrm{~mm}$, the ratio of the time scale for heat conduction on the macroscale of the snowpack to the time scale for heat conduction on the microscale is on the order of $10^{6}$, which suggests that macroscale thermal equilibrium between the ice and humid air constituents is a good assumption. Moreover, the large separation of scales in the time domain is consistent with the discussion of Auriault et al. (2009) regarding separation of time scales necessary for homogenization.

The assumption of uniform constituent temperatures at the macroscale should not be confused with the local (microscale) temperature. Under a macroscale temperature gradient, local constituent temperatures in the interior of the RVE differ due to different thermal conductivities of the ice and humid air. Further, temperature gradients within individual constituents are also present at the microscale. A warmer ice grain is separated from a colder ice grain by pore space, for example. These temperature differentials drive the mass transfer process at the microscale. Again, an excellent insight into microscale thermal behavior is provided in Fig. 4 of Pinzer et al. (2012).

Thermal equilibrium of the ice and humid air constituents at the macroscale allows the constituent energy equations, (Eqs. 47 and 56), to be added together to yield an energy equation for snow with a single temperature as

$\left(\phi_{\mathrm{ha}} \gamma_{\mathrm{ha}} C_{\mathrm{ha}}^{\mathrm{V}}+\phi_{\mathrm{i}} \gamma_{\mathrm{i}} C_{\mathrm{i}}^{\mathrm{V}}\right) \frac{\partial \theta}{\partial t}=\nabla \cdot\left(k_{\mathrm{s}}^{\mathrm{eff}} \nabla \theta\right)+\hat{c} u_{\mathrm{sg}}$,

where $\theta$ is the temperature of the snow. Notably, the constituent energy supply terms sum to zero in the energy equation for snow and the volume averaged constituent effective thermal conductivities have been absorbed into an effective thermal conductivity for snow, $k_{\mathrm{s}}^{\text {eff }}$, as

$k_{\mathrm{s}}^{\mathrm{eff}}=\phi_{\mathrm{i}} k_{\mathrm{i}}^{\mathrm{eff}}+\phi_{\mathrm{ha}} k_{\mathrm{ha}}^{\mathrm{eff}}$.

While the effective thermal conductivities, $k_{\mathrm{i}}^{\text {eff }}$ and $k_{\mathrm{ha}}^{\mathrm{eff}}$, are never computed, it would be important to do so if one wanted to study non-equilibrium constituent temperatures on a short time scale with a mixture theory.

One can make a direct connection of $k_{i}^{\text {eff }}$ and $k_{\text {ha }}^{\text {eff }}$ with the work of Calonne et al. (2014a). Specifically, the tensorial form of the effective thermal conductivity for snow is defined in Eq. (25) of Calonne et al. (2014a) as

$\boldsymbol{k}_{\mathrm{s}}^{\mathrm{eff}}=\frac{1}{|V|}\left(\int_{V a} k_{\mathrm{a}}\left(\nabla \boldsymbol{t}_{\mathrm{a}}+\boldsymbol{I}\right) \mathrm{d} V+\int_{V i} k_{\mathrm{i}}\left(\boldsymbol{\nabla} \boldsymbol{t}_{\mathrm{i}}+\boldsymbol{I}\right) \mathrm{d} V\right)$,

where $\boldsymbol{t}_{\alpha}$ characterizes the temperature fluctuation in constituent $\alpha$ and $\boldsymbol{I}$ is the identity tensor.

The above equation may be rearranged as

$\boldsymbol{k}_{\mathrm{s}}^{\mathrm{eff}}=\phi_{\mathrm{a}} \frac{1}{\left|V_{\mathrm{a}}\right|} \int_{V a} k_{\mathrm{a}}\left(\nabla \boldsymbol{t}_{\mathrm{a}}+\boldsymbol{I}\right) \mathrm{d} V+\phi_{\mathrm{i}} \frac{1}{\left|V_{\mathrm{i}}\right|} \int_{V i} k_{\mathrm{i}}\left(\nabla \boldsymbol{t}_{\mathrm{i}}+\boldsymbol{I}\right) \mathrm{d} V$.

Comparing Eqs. (62) and (64) provides a clear mathematical interpretation of $k_{\mathrm{i}}^{\text {eff }}$ and $k_{\mathrm{ha}}^{\text {eff }}$ as

$\boldsymbol{k}_{\mathrm{ha}}^{\mathrm{eff}}=\frac{1}{\left|V_{\mathrm{a}}\right|} \int_{V a} k_{\mathrm{a}}\left(\nabla \boldsymbol{t}_{\mathrm{a}}+\boldsymbol{I}\right) \mathrm{d} V$, 
and

$\boldsymbol{k}_{\mathrm{i}}^{\mathrm{eff}}=\frac{1}{\left|V_{\mathrm{i}}\right|} \int_{V i} k_{\mathrm{i}}\left(\nabla \boldsymbol{t}_{\mathrm{i}}+\boldsymbol{I}\right) \mathrm{d} V$.

Finally, recent research work has shown the effective thermal conductivity of snow to be anisotropic, see for example Schertzer and Adams (2011) and Riche and Schneebeli (2013). We avoid this complexity at present as it becomes a non-issue for the 1-D heat and mass transfer theory developed subsequently.

To summarize, the governing equations for heat and water vapor transfer in snow are given by Eqs. (42) and (61). These equations are identical to macroscale equations developed by Calonne et al. (2014a) through a description at the pore scale using the homogenization of multiple scale expansions. The equality is best shown by multiplying the right-hand side of Eq. (20) in Calonne by $\left(\rho_{\mathrm{i}} / \rho_{\mathrm{i}}\right)$ and relabeling $\left(L_{\mathrm{sg}} / \rho_{\mathrm{i}}\right)$ as $u_{\mathrm{sg}}$, resulting in Eq. (61) of the present paper. Equation (42) is already identical in form to Eq. (21) of Calonne et al. (2014a).

While the equations of Foslien (1994) and Calonne et al. (2014a) governing the macroscale response of heat and mass transfer in snow are identical, the emphasis of Calonne's work is on upscaling, whereas the present paper focuses on solutions of the macroscale behavior. We also address similarities and differences in the calculation of effective thermal conductivity and the effective diffusion coefficient for snow, critical parameters affecting macroscale sublimation and deposition rates in a snow cover.

\subsection{Saturated vapor density at the macroscale}

A physical interpretation of the mass supply term, $\hat{c}$, is the mass rate at which water vapor is condensing to form ice per unit volume of snow. Hobbs (1974) provides an expression for the condensation of water vapor to ice driven by a difference in the vapor pressure and the saturated vapor pressure over ice, $\left(p-p^{\text {sat }}\right)$, as

$$
\frac{\alpha_{\mathrm{c}} m_{\mathrm{mol}}\left(p-p^{\mathrm{sat}}\right)}{\left(2 \pi m_{\mathrm{mol}} \Omega \theta\right)^{1 / 2}} \mathrm{~kg} \mathrm{~m}^{-2} \mathrm{~s}^{-1},
$$

where $m_{\mathrm{mol}}$ is the mass per molecule of water, $\Omega$ is Boltzman's constant, and $\alpha_{\mathrm{c}}$ is the condensation coefficient.

Multiplying the above expression by the specific surface area of snow, $\xi$, and utilizing the ideal gas law for water vapor provides an explicit expression for the mass supply driven by a difference in vapor density given by

$\hat{c}=\frac{\xi R \theta \alpha_{\mathrm{c}} m_{\mathrm{mol}}\left(\gamma_{\mathrm{v}}-\gamma_{\mathrm{v}}^{\mathrm{sat}}\right)}{\left(2 \pi m_{\mathrm{mol}} \Omega \theta\right)^{1 / 2}}$.

In the absence of diffusion, Eq. (67) can be combined with the mass balance equation (Eq. 42) for the water vapor as

$\phi_{\mathrm{v}} \frac{\partial \gamma_{\mathrm{v}}}{\partial t}=\frac{\xi R \theta \alpha_{\mathrm{c}} m_{\mathrm{mol}}\left(\gamma_{\mathrm{v}}-\gamma_{\mathrm{v}}^{\mathrm{sat}}\right)}{\left(2 \pi m_{\mathrm{mol}} \Omega \theta\right)^{1 / 2}}$
If the saturated vapor density over the ice is held constant, the time for the vapor density difference between the pore density and the saturated vapor density to become $0.1 \%$ of the initial density difference can be computed. Delaney et al. (1964) measured the condensation coefficient, $\alpha_{\mathrm{c}}$, of ice to be 0.0144 for temperatures between 271 and $260 \mathrm{~K}$. For a snow density of $200 \mathrm{~kg} \mathrm{~m}^{-3}$ and a specific surface area of $1400 \mathrm{~m}^{-1}$, the time for the vapor density in the pore to reach equilibrium is approximately $1.1 \times 10^{-3} \mathrm{~s}$. Hence, the vapor density in a pore can be assumed to be the saturated vapor density throughout the process of heat and mass transfer occurring at the macroscale where the time scale of interest is on the order of hours or days.

The knowledge that the vapor density may be assumed saturated in a macroscale analysis affords a critical simplification in the mixture theory analysis in that a constitutive law for the mass supply is no longer needed. Instead, the mass supply is computed from Eq. (42) by noting the water vapor is always saturated at the snow temperature, leading to

$$
\hat{c}=\nabla \cdot\left(D_{\mathrm{s}}^{\mathrm{eff}} \nabla \gamma_{\mathrm{v}}^{\mathrm{sat}}\right)-\phi_{\mathrm{ha}} \frac{\partial \gamma_{\mathrm{v}}^{\mathrm{sat}}}{\partial t}
$$

We emphasize that Eq. (67) is not utilized in the snowpack modeling of water vapor deposition and sublimation found in Sect. 6 as it is replaced by Eq. (69).

\subsection{Formulation summary}

At this point, we restrict the development to a 1-D model and write the energy equation, Eq. (61), as

$\left(\phi_{\mathrm{ha}} \gamma_{\mathrm{ha}} C_{\mathrm{ha}}^{\mathrm{V}}+\phi_{\mathrm{i}} \gamma_{\mathrm{i}} C_{\mathrm{i}}^{\mathrm{V}}\right) \frac{\partial \theta}{\partial t}=\frac{\partial}{\partial x}\left(k_{\mathrm{s}}^{\mathrm{eff}} \frac{\partial \theta}{\partial x}\right)+\hat{c} u_{\mathrm{sg}}$.

The mass supply equation, Eq. (69), representing phase changes due to condensation or sublimation assumes the 1-D form

$\hat{c}=\frac{\partial}{\partial x}\left(D_{\mathrm{s}}^{\mathrm{eff}} \frac{\partial \gamma_{\mathrm{v}}^{\mathrm{sat}}}{\partial x}\right)-\phi_{\mathrm{ha}} \frac{\partial \gamma_{\mathrm{v}}^{\mathrm{sat}}}{\partial t}$.

The saturated vapor density may be expressed as purely a function of temperature (Dorsey, 1968) leading to

$\frac{\partial \gamma_{\mathrm{v}}^{\text {sat }}}{\partial x}=\frac{\mathrm{d} \gamma_{\mathrm{v}}^{\text {sat }}}{\mathrm{d} \theta} \frac{\partial \theta}{\partial x}$ and $\frac{\partial \gamma_{\mathrm{v}}^{\mathrm{sat}}}{\partial t}=\frac{\mathrm{d} \gamma_{\mathrm{v}}^{\mathrm{sat}}}{\mathrm{d} \theta} \frac{\partial \theta}{\partial t}$.

Noting the above, the mass supply equation, Eq. (71), is expressed as

$\hat{c}=\frac{\partial}{\partial x}\left(D_{\mathrm{s}}^{\mathrm{eff}} \frac{\mathrm{d} \gamma_{\mathrm{v}}^{\mathrm{sat}}}{\mathrm{d} \theta} \frac{\partial \theta}{\partial x}\right)-\phi_{\mathrm{ha}} \frac{\mathrm{d} \gamma_{\mathrm{v}}^{\mathrm{sat}}}{\mathrm{d} \theta} \frac{\partial \theta}{\partial t}$.

Finally, substituting Eq. (72) into Eq. (70) leads to a single partial differential equation governing the energy balance for 
snow given by

$$
\begin{aligned}
& \left(\phi_{\mathrm{ha}} \gamma_{\mathrm{ha}} C_{\mathrm{ha}}^{\mathrm{V}}+\phi_{\mathrm{i}} \gamma_{\mathrm{i}} C_{\mathrm{i}}^{\mathrm{V}}+u_{\mathrm{sg}} \phi_{\mathrm{ha}} \frac{\mathrm{d} \gamma_{\mathrm{v}}^{\mathrm{sat}}}{\mathrm{d} \theta}\right) \frac{\partial \theta}{\partial t} \\
& =\frac{\partial}{\partial x}\left(k_{\mathrm{s}}^{\mathrm{con}+\mathrm{d}} \frac{\partial \theta}{\partial x}\right),
\end{aligned}
$$

where

$k_{\mathrm{s}}^{\mathrm{con}+\mathrm{d}}=k_{\mathrm{s}}^{\mathrm{eff}}+u_{\mathrm{sg}} D_{\mathrm{s}}^{\mathrm{eff}} \frac{\mathrm{d} \gamma_{\mathrm{v}}^{\mathrm{sat}}}{\mathrm{d} \theta}$.

The thermal conductivity $k_{\mathrm{s}}^{\mathrm{con}+\mathrm{d}}$ is the apparent effective thermal conductivity of snow that accounts for heat conduction, $k_{\mathrm{s}}^{\text {eff }}$, as well as energy transfer due to water vapor diffusion.

Rather than combining Eqs. (70) and (72) and solving Eq. (73), it is more insightful to solve Eqs. (70) and (72) separately. Retaining a separate equation for the mass supply allows one to quantify macroscale deposition and sublimation rates, a fundamental objective of the theory developed herein.

\section{Evaluation of the effective thermal conductivity and the effective diffusion coefficient for snow}

Solution of the energy equation (Eq. 70) and the mass balance equation (Eq. 72) requires knowledge of macroscale parameters for effective thermal conductivity as well as the effective diffusion coefficient for snow. Calonne et al. (2011) and Riche and Schneebeli (2013) have performed extensive numerical studies using finite element analysis coupled with $\mathrm{X}$-ray tomography to quantify the effective thermal conductivity for snow as a function of density at a fixed temperature. Calonne et al. (2011) also provide effective thermal conductivity predictions at two separate temperatures. Pinzer et al. (2012) and Christon et al. (1994) performed numerical studies aimed at determining the effective diffusion coefficient for snow. Calonne et al. (2014a) also used finite element micromechanics to predict an effective diffusion coefficient for snow although the specific numerical approach to evaluate this parameter followed a fundamentally approach.

Regardless of the parameter being studied, a drawback of microscale finite element analysis (micromechanics) is that the results provide heat and mass transfer properties at a single temperature and density. Hence, a complete characterization of these parameters as a function of density and temperature requires a significant number of micromechanics solutions at multiple densities and temperatures followed by a curve-fitting exercise.

Rather than relying on finite element micromechanics solutions, we present an analytical approach developed by Foslien (1994) to predict values for the effective thermal conductivity and the effective diffusion coefficient of snow. Foslien's model has several attractive features including the following:

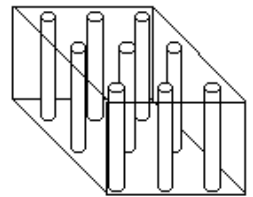

(a) Pore Microstructure

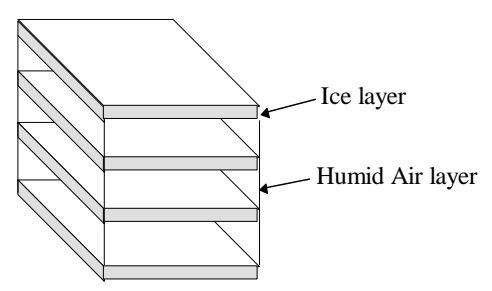

(b) Lamellae Microstructure

Figure 2. Idealized microstructures used to model heat and mass transfer in parallel (a) and series (b).

- there is excellent correlation with cited finite element results for effective thermal conductivity and effective diffusion coefficient for snow;

- density effects are explicitly introduced in the analytical model through volume fractions, while temperature effects appear implicitly through thermal conductivity properties for ice and air;

- the effects of mass diffusion on the energy flux are explicit and the relative influence on the energy flux is readily determined;

- the model provides self-consistent results for effective thermal conductivity and effective diffusion coefficient for snow for the limiting cases of air and ice;

- the model is developed from first principles of heat and mass transfer applied to simple microstructures and contains no empirical coefficients of adjustment.

Foslien's development begins by formulating microscale heat and mass transfer models for classic microstructures consisting of ice and humid air acting in parallel and series, respectively. Heat and mass transfer properties for snow are then proposed using arguments from quantitative stereology.

Figure 2a shows an ice matrix with humid air pores in parallel to an applied temperature gradient. In this pore microstructure, energy is transferred in parallel through the snowpack. The energy fluxes for the ice $\left(\boldsymbol{q}_{\mathrm{i}}\right)$ and humid air $\left(\boldsymbol{q}_{\text {ha }}\right)$ constituents are simply added together to obtain the total energy flux through the snowpack. Because the thermal conductivity of ice is roughly 100 times larger than for the humid air, the ice phase plays a dominant role in heat transfer for this microstructure. 
The second microstructure studied, referred to as a lamellae microstructure, consisted of ice and humid air layers oriented perpendicular to the energy flux (Fig. 2b). In this case, energy flows in series through the respective layers. Hence, the energy flux in the humid air constituent must equal the energy flux through the ice constituent. An interesting feature of mass transfer in the lamellae microstructure is that diffusion via the "hand to hand" model described by Yosida (1955) is naturally present and accounted for in the development. Specifically, diffusion is enhanced as the total path length for diffusion is reduced by the ice layer which acts as both a source and sink for water vapor.

The two microstructures studied by Foslien (1994) were first considered by de Quervain (1963) and produce two very different heat and mass transfer results that are believed to represent the extremes possible for ice and humid air mixtures.

\subsection{Pore microstructure}

Foslien's heat and mass transfer analysis of the pore microstructure begins by writing energy flux expressions for the ice and humid air constituents at the macroscale. The energy flux of the ice is attributed to heat conduction, leading to

$q_{\mathrm{i}}=-k_{\mathrm{i}} \frac{\partial \theta}{\partial x}$.

The energy flux of the humid air is attributed to conduction of the humid air and the mass flux of water vapor. Following Bird and Lightfoot (1960) we can write

$q_{\mathrm{ha}}=-k_{\mathrm{ha}} \frac{\partial \theta}{\partial x}-u_{\mathrm{sg}} D_{\mathrm{v}-\mathrm{a}} \frac{\mathrm{d} \gamma_{\mathrm{v}} \mathrm{sat}}{\mathrm{d} \theta} \frac{\partial \theta}{\partial x}$.

The energy flux of the pore microstructure is introduced as

$q_{\text {pore }}=-k_{\text {pore }} \frac{\partial \theta}{\partial x}$.

Energy transfer in the pore microstructure occurs in parallel and the energy flux is simply the volume average of the energy fluxes of the ice and humid air leading to

$k_{\text {pore }}=\phi_{\mathrm{i}} k_{\mathrm{i}}+\phi_{\mathrm{ha}} k_{\mathrm{ha}}+\phi_{\mathrm{ha}} u_{\mathrm{sg}} D_{\mathrm{v}-\mathrm{a}} \frac{\mathrm{d} \gamma_{\mathrm{v}} \mathrm{sat}}{\mathrm{d} \theta}$.

\subsection{Lamellae microstructure}

The discontinuous nature of the lamellae microstructure in the direction of interest introduces a complexity in the spatial gradients, as the constituent gradients must be defined with respect to a differential length, $\mathrm{d} x_{\alpha}$. Hence the constituent energy fluxes assume the form

$q_{\mathrm{i}}=-k_{\mathrm{i}} \frac{\partial \theta}{\partial x_{\mathrm{i}}}$,

and

$q_{\mathrm{ha}}=-k_{\mathrm{ha}} \frac{\partial \theta}{\partial x_{\mathrm{ha}}}-u_{\mathrm{sg}} D_{\mathrm{v}-\mathrm{a}} \frac{\mathrm{d} \gamma_{\mathrm{v}^{\mathrm{sat}}}}{\mathrm{d} \theta} \frac{\partial \theta}{\partial x_{\mathrm{ha}}}$.

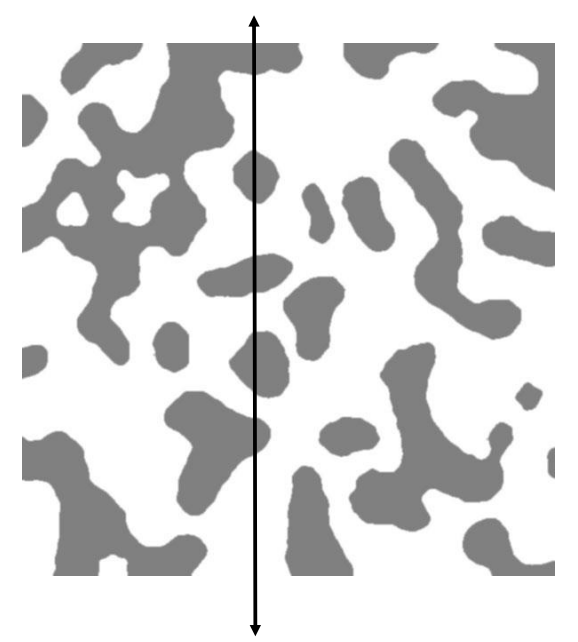

\section{1-D Heat Transfer}

Figure 3. Test line through a snow surface section showing 1-D heat and mass transfer at the microscale.

The average temperature gradient expressed in terms of the macroscale coordinate $x$ is given by

$\frac{\partial \theta}{\partial x}=\phi_{\mathrm{i}} \frac{\partial \theta}{\partial x_{\mathrm{i}}}+\phi_{\mathrm{ha}} \frac{\partial \theta}{\partial x_{\mathrm{ha}}}$.

The energy flux through the lamellae microstructure is introduced as

$q_{\text {lam }}=-k_{\operatorname{lam}} \frac{\partial \theta}{\partial x}$.

Equations (79)-(82) may be combined to arrive at

$k_{\text {lam }}=\frac{k_{\mathrm{i}}\left(k_{\mathrm{ha}}+u_{\mathrm{sg}} D_{\mathrm{v}-\mathrm{a}} \frac{\mathrm{d} \gamma_{\mathrm{v}}^{\text {sat }}}{\mathrm{d} \theta}\right)}{\phi_{\mathrm{i}}\left(k_{\mathrm{a}}+u_{\mathrm{sg}} D_{\mathrm{v}-\mathrm{a}} \frac{\mathrm{d} \gamma_{\mathrm{v}}^{\mathrm{sat}}}{\mathrm{d} \theta}\right)+\phi_{\mathrm{ha}} k_{\mathrm{i}}}$.

\subsection{Snow properties}

The energy flux for snow accounts for heat conduction as well as energy transfer due to water vapor diffusion. From Eqs. (73) and (74), the energy flux may be identified as

$q_{\mathrm{s}}=-\left(k_{\mathrm{s}}^{\mathrm{eff}}+u_{\mathrm{sg}} D_{\mathrm{s}}^{\mathrm{eff}} \frac{\mathrm{d} \gamma_{\mathrm{v}}^{\mathrm{sat}}}{\mathrm{d} \theta}\right) \frac{\partial \theta}{\partial x}$.

Foslien (1994) proposed an energy flux for snow that includes energy transfer due to heat conduction and mass diffusion as

$q_{\mathrm{s}}=\phi_{\mathrm{i}} q_{\mathrm{pore}}+\phi_{\mathrm{ha}} q_{\mathrm{lam}}$.

Justification for Eq. (85) is provided by considering a snow surface section as shown in Fig. 3. When a test line is arbitrarily drawn through the surface section, a fraction of the total 
length will pass through the ice constituent, and the remainder will pass through the humid air constituent. If one imagines a 1-D heat transfer occurring along the test line, heat transfer through the ice phase is dominated by the pore microstructure where the thermal conductivity of ice is nearly 100 times that of air. In contrast, anytime the test line passes through the humid air constituent, heat transfer would be dominated by the lamellae microstructure. Using the lineal fraction as the weighted behavior of the thermal conductivity and recognizing the lineal fraction is identical to the volume fraction under conditions of isotropy (Underwood, 1970) leads directly to Eq. (85).

Combining Eqs. (77)-(78) and Eqs. (82)-(83) with Eq. (85) leads to an expression for the energy flux of snow given by

$q_{\mathrm{s}}=-\left[\phi_{\mathrm{i}}\left(\phi_{\mathrm{ha}} k_{\mathrm{ha}}+\phi_{\mathrm{i}} k_{\mathrm{i}}\right)+\phi_{\mathrm{ha}}\left(\frac{k_{\mathrm{i}} k_{\mathrm{ha}}}{\phi_{\mathrm{i}}\left(k_{\mathrm{ha}}+u_{\mathrm{sg}} D_{\mathrm{v}-\mathrm{a}} \frac{\mathrm{d} \gamma_{\mathrm{v}}^{\mathrm{sat}}}{\mathrm{d} \theta}\right)+\phi_{\mathrm{ha}} k_{\mathrm{i}}}\right)\right]$ $\frac{\partial \theta}{\partial x}-\left\{\phi_{\mathrm{i}}\left(\phi_{\mathrm{ha}} D_{\mathrm{v}-\mathrm{a}}\right)+\phi_{\mathrm{ha}}\left(\frac{k_{\mathrm{i}} D_{\mathrm{v}-\mathrm{a}}}{\phi_{\mathrm{i}}\left(k_{\mathrm{ha}}+u_{\mathrm{sg}} D_{\mathrm{v}--\mathrm{a}} \frac{\mathrm{d} \gamma_{\mathrm{v}}^{\mathrm{sat}}}{\mathrm{d} \theta}\right)+\phi_{\mathrm{ha}} k_{\mathrm{i}}}\right)\right\}$

$u_{\mathrm{sg}} \frac{\mathrm{d} \gamma_{\mathrm{v}}^{\mathrm{sat}}}{\mathrm{d} \theta} \frac{\partial \theta}{\partial x}$.

Motivated by the functional forms of Eqs. (84) and (86), we define the effective thermal conductivity and effective diffusion coefficient as

$$
\begin{aligned}
k_{\mathrm{s}}^{\mathrm{eff}}= & \phi_{\mathrm{i}}\left(\phi_{\mathrm{ha}} k_{\mathrm{ha}}+\phi_{\mathrm{i}} k_{\mathrm{i}}\right)+\phi_{\mathrm{ha}} \\
& \left(\frac{k_{\mathrm{i}} k_{\mathrm{ha}}}{\phi_{\mathrm{i}}\left(k_{\mathrm{ha}}+u_{\mathrm{sg}} D_{\mathrm{v}-\mathrm{a}} \frac{\mathrm{d} \gamma_{\mathrm{v}}^{\text {sat }}}{\mathrm{d} \theta}\right)+\phi_{\mathrm{ha}} k_{\mathrm{i}}}\right),
\end{aligned}
$$

and

$$
\begin{aligned}
D_{\mathrm{s}}^{\mathrm{eff}}= & \phi_{\mathrm{i}}\left(\phi_{\mathrm{ha}} D_{\mathrm{v}-\mathrm{a}}\right)+\phi_{\mathrm{ha}} \\
& \left(\frac{k_{\mathrm{i}} D_{\mathrm{v}-\mathrm{a}}}{\phi_{\mathrm{i}}\left(k_{\mathrm{ha}}+u_{\mathrm{sg}} D_{\mathrm{v}-\mathrm{a}} \frac{\mathrm{d} \gamma_{\mathrm{v}}^{\mathrm{sat}}}{\mathrm{d} \theta}\right)+\phi_{\mathrm{ha}} k_{\mathrm{i}}}\right) .
\end{aligned}
$$

Despite the presence of the binary diffusion coefficient of water vapor in air in the expression for $k_{\mathrm{s}}^{\mathrm{eff}}$, it should be emphasized that the result given in Eq. (87) represents the effective thermal conductivity for snow as predicted by the analytical model. Similarly, constituent thermal conductivity parameters appear in the equation for the effective diffusion coefficient of snow, $D_{\mathrm{s}}^{\text {eff }}$. These results are a consequence of a direct application of heat and mass transfer principles for the lamellae microstructure - the parameters of thermal conductivity and diffusion simply do not separate at the macroscale for this microstructure.

A good deal of clarity in the physical interpretation of $k_{\mathrm{s}}^{\text {eff }}$ and $D_{\mathrm{s}}^{\text {eff }}$ may be achieved through an order of magnitude analysis of the various terms in Eqs. (87) and (88). To begin, for the range of temperatures of interest, one may show $k_{\mathrm{ha}}$ and $\left(u_{\mathrm{sg}} D_{\mathrm{v}-\mathrm{a}} \frac{\mathrm{d} \gamma_{\mathrm{v}}^{\text {sat }}}{\mathrm{d} \theta}\right)$ are of the same order of magnitude. Now rearrange Eqs. (87) and (88) by dividing numerator and denominator of the last term in each by $k_{\mathrm{i}}$, leading to

$$
k_{\mathrm{s}}^{\text {eff }}=\phi_{\mathrm{i}}\left(\phi_{\mathrm{ha}} k_{\mathrm{ha}}+\phi_{\mathrm{i}} k_{\mathrm{i}}\right)+\phi_{\mathrm{ha}}\left(\frac{k_{\mathrm{ha}}}{\phi_{\mathrm{i}}\left[\frac{k_{\mathrm{ha}}+u_{\mathrm{sg}} D_{\mathrm{v}-\mathrm{a}} \frac{\mathrm{d} \gamma_{\mathrm{v}}^{\mathrm{sat}}}{\mathrm{d} \theta}}{k_{\mathrm{i}}}\right]+\phi_{\mathrm{ha}}}\right),
$$

and

$$
D_{\mathrm{s}}^{\mathrm{eff}}=\phi_{\mathrm{i}}\left(\phi_{\mathrm{ha}} D_{\mathrm{v}-\mathrm{a}}\right)+\phi_{\mathrm{ha}}\left(\frac{D_{\mathrm{v}-\mathrm{a}}}{\phi_{\mathrm{i}}\left[\frac{k_{\mathrm{ha}}+u_{\mathrm{sg}} D_{\mathrm{v}-\mathrm{a}} \frac{\mathrm{d} \gamma_{\mathrm{v}}^{\mathrm{sat}}}{\mathrm{d} \theta}}{k_{\mathrm{i}}}\right]+\phi_{\mathrm{ha}}}\right) .
$$

The value of the thermal conductivity of ice is on the order of 100 times that of the term $\left(k_{\mathrm{ha}}+u_{\mathrm{sg}} D_{\mathrm{v}-\mathrm{a}} \frac{\mathrm{d} \gamma_{\mathrm{v}}^{\mathrm{sat}}}{\mathrm{d} \theta}\right)$. Therefore, neglecting the term in square brackets in the above expressions for $k_{\mathrm{s}}^{\mathrm{eff}}$ and $D_{\mathrm{s}}^{\mathrm{eff}}$ leads to

$k_{\mathrm{s}}^{\mathrm{eff}}=\phi_{\mathrm{i}}\left(\phi_{\mathrm{ha}} k_{\mathrm{ha}}+\phi_{\mathrm{i}} k_{\mathrm{i}}\right)+k_{\mathrm{ha}}$,

and

$D_{\mathrm{s}}^{\mathrm{eff}}=\phi_{\mathrm{i}} \phi_{\mathrm{ha}} D_{\mathrm{v}-\mathrm{a}}+D_{\mathrm{v}-\mathrm{a}}$.

Equations (91) and (92) reveal a desirable consistency in terms. Specifically, the effective thermal conductivity of snow depends only on the thermal conductivities of ice and humid air, respectively, while the effective diffusion coefficient for snow depends only on the binary coefficient of water vapor in air. Hence, the thermal conductivity and diffusion expressions decouple from one another.

Owing to the "clean" nature of the simplified forms for $k_{\mathrm{s}}^{\mathrm{eff}}$ and $D_{\mathrm{s}}^{\text {eff }}$, one might be tempted to use them at all times. That approach is, indeed, valid for the effective thermal conductivity as the simplified effective thermal conductivity curve is nearly identical to the original proposed by Foslien. However, important differences arise in the diffusion predictions.

Figure 4 shows the effective diffusion curves predicted by Eqs. (88) and (92), respectively. The two curves are identical over a wide range of densities from approximately 0 to $400 \mathrm{~kg} \mathrm{~m}^{-3}$. As the curves deviate at higher densities, the original form proposed by Foslien is necessary to drive $D_{\mathrm{s}}^{\text {eff }}$ to the known limiting value of zero for solid ice. The consistency of Foslien's model is impressive in this regard.

There is yet another physically pleasing aspect of Foslien's model for the effective diffusion coefficient for snow. Using the simplified form of Eq. (92), one can write the effective diffusion coefficient as

$D_{\mathrm{s}}^{\mathrm{eff}}=\phi_{\mathrm{i}}\left(\phi_{\mathrm{ha}} D_{\mathrm{v}-\mathrm{a}}\right)+\phi_{\mathrm{ha}}\left(\frac{D_{\mathrm{v}-\mathrm{a}}}{\phi_{\mathrm{ha}}}\right)$. 


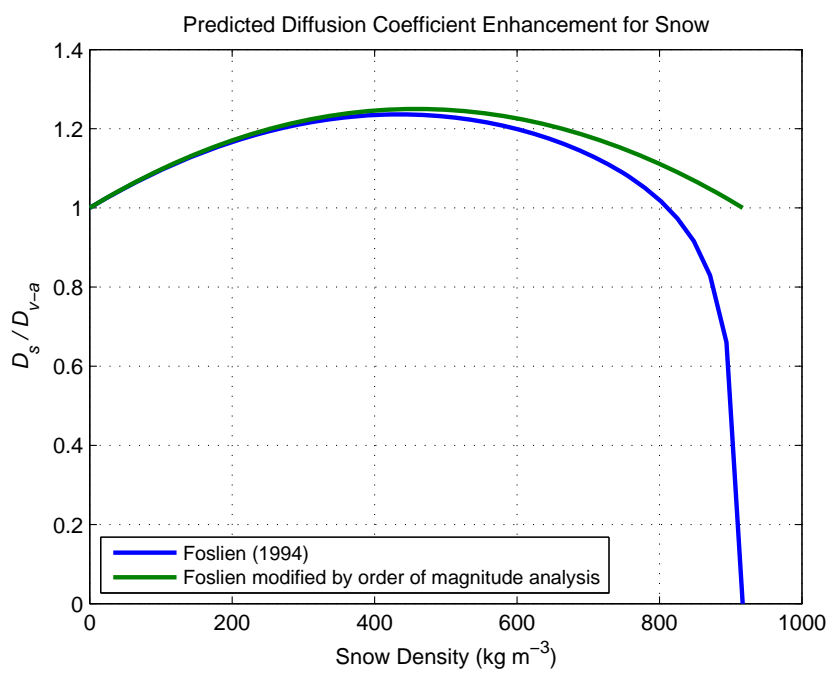

Figure 4. Predicted diffusion coefficient enhancement for snow using the original model of Foslien and a simplified model based on an order of magnitude analysis.

The leading volume fraction in each of the terms in the above equation is attributed to the volume fraction weighting of the snow model proposed by Foslien, allowing us to identify effective diffusion coefficients for the pore and lamellae microstructures as

$D_{\text {pore }}=\left(\phi_{\mathrm{ha}} D_{\mathrm{v}-\mathrm{a}}\right)$ and $D_{\mathrm{lam}}=\left(\frac{D_{\mathrm{v}-\mathrm{a}}}{\phi_{\mathrm{ha}}}\right)$.

In the case of the effective diffusion coefficient for the pore microstructure, the humid air volume fraction leads $D_{\mathrm{v}-\mathrm{a}}$. The interpretation of $\phi_{\text {ha }}$ is quite clear, as the ice phase acts as a blockage and limits the amount of area for humid air mass transport to occur.

The influence of the ice phase on the effective diffusion of water vapor is fundamentally different for the lamellae microstructure compared to the pore microstructure. First, the ice does not act as a blockage of diffusion paths in the lamellae microstructure as it does in the pore microstructure. Secondly, the ice phase actually enhances water vapor diffusion in the lamellae microstructure by shortening the pathway needed to travel via the "hand to hand" mechanism described by Yosida (1955). For example, given an ice volume fraction of 0.5 , one would expect the diffusion coefficient of the lamellae microstructure to be double that found in humid air as water vapor would only have to travel half the distance compared to the distance traveled in humid air alone. Taken collectively, these factors suggest the influence of $\phi_{\text {ha }}$ on the diffusion coefficient $D_{\text {lam }}$ should scale as $\left(1 / \phi_{\text {ha }}\right)$, precisely as Foslien's model predicts.

While the idealized microstructures utilized by Foslien are not representative of the complex microstructure of snow, the mass transfer mechanisms described above that are associated with each microstructure are clearly present in snow. Im-

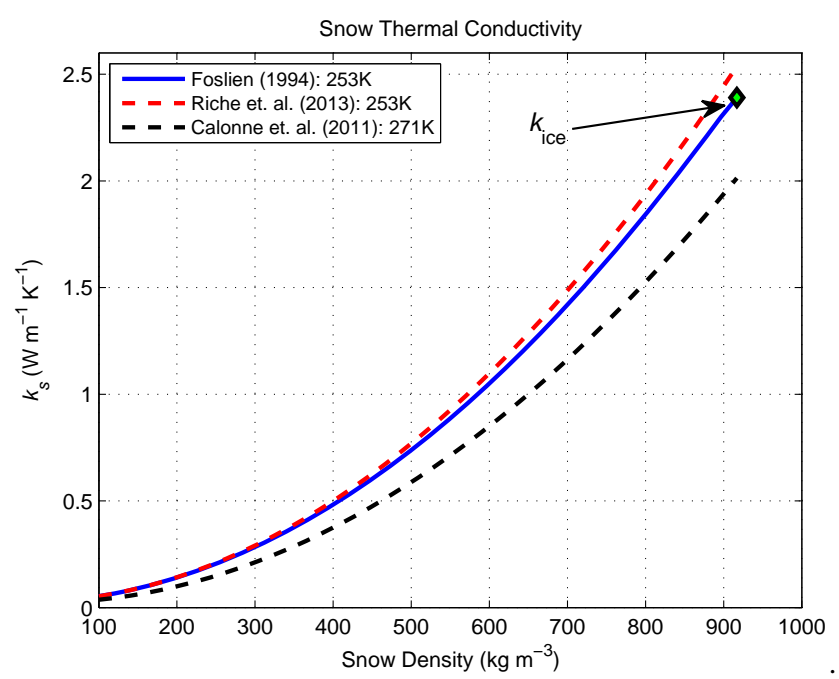

Figure 5. Thermal conductivity analytical prediction of Foslien (1994) vs. finite element predictions of Calonne et al. (2011) and Riche and Schneebeli (2013).

portantly, the proposed diffusion model captures these mechanisms.

\subsubsection{Effective thermal conductivity}

Calonne et al. (2011) and Riche and Schneebeli (2013) provide curve fits of snow effective thermal conductivity as a function of density based on their finite element micromechanics analyses. Calonne's data included analysis of crystal structures of all types, while Riche's data were limited to depth hoar and faceted crystals which produce higher thermal conductivities in the direction of interest (normal to the ground).

Figure 5 provides the predictions of Eq. (87) for a temperature of $253 \mathrm{~K}$ against the curve fits of Calonne et al. (2011) and Riche and Schneebeli (2013). The correlation of the analytical model is excellent as the model virtually tracks the numerical results of Riche and Schneebeli (2013) whose data were also generated at $253 \mathrm{~K}$. Fosliens's predicted curve at $271 \mathrm{~K}$ shifts downward toward the curve generated by Calonne et al. (2011), also generated at $271 \mathrm{~K}$, but remains well within the bounds of both curves generated through finite element analysis of real microstructures. Furthermore, the most significant deviation of the analytical model occurs at a density for solid ice where Foslien's model predicts the self-consistent correct result of thermal conductivity for ice.

Changes in effective thermal conductivity as a function of temperature were observed by Calonne et al. (2011) for temperatures of 271 and $203 \mathrm{~K}$, respectively. Figure 6 shows the effective thermal conductivity line predicted by Foslien along with the numerical micromechanics predictions of Calonne et al. (2011). Excellent correlation of the analytical model and the finite element analyses is again observed. 


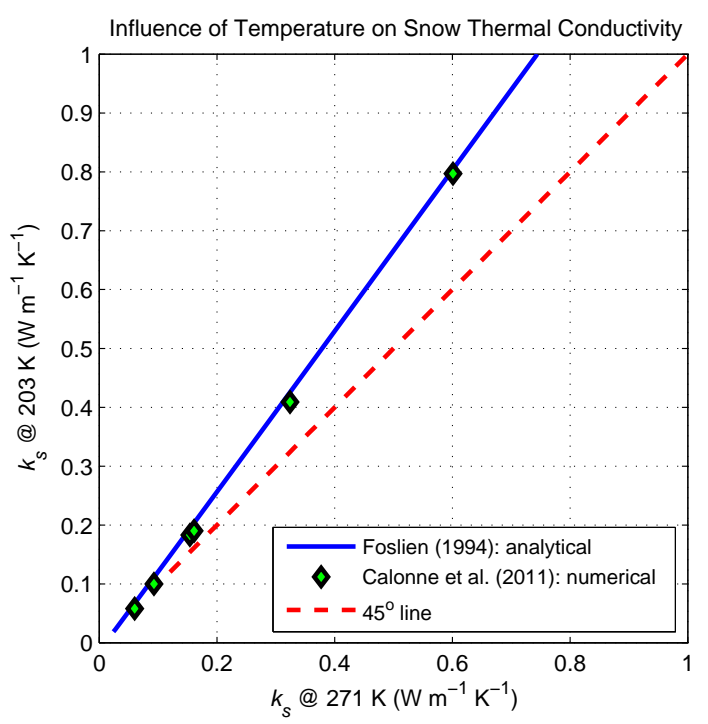

Figure 6. The influence of temperature on thermal conductivity as predicted by the model of Foslien (1994) and Calonne et al. (2011) through finite element analysis.

Figures 5 and 6 demonstrate the proposed model for effective thermal conductivity of snow does an excellent job of quantifying $k_{\mathrm{s}}^{\text {eff }}$ as a function of density and temperature. However, the complex nature of the microstructure of snow and the inability to relate the geometric structure to material properties results in significant scatter in the effective thermal conductivity properties when viewed solely as a function of density.

Calonne et al. (2014b) performed a series of experiments on snow metamorphism showing a variability of snow effective thermal conductivity under near constant density conditions. The experiments also showed the anisotropic properties of the effective thermal conductivity tensor increased during metamorphism. Löwe et al. (2013) developed a microstructural parameter that can be used to reduce the scatter in effective thermal conductivity predictions based on density alone. The parameter also provides a means of incorporating anisotropic behavior of effective thermal conductivity. It would be advantageous to refine the effective thermal conductivity model of Eq. (87) to include microstructural effects other than density. Such a refinement may be particularly important when extending the current 1-D analysis to higher spatial dimensions needed for modeling heat and mass transfer in complex terrain.

\subsubsection{Effective diffusion coefficient}

The value of the effective diffusion coefficient, $D_{\mathrm{s}}^{\text {eff }}$, for snow has a long history of dispute. Giddings and LaChapelle (1962) claimed $D_{\mathrm{s}}^{\text {eff }}$ to be less than the diffusion coefficient of water vapor in air due to the fact that ice grains interfere with diffusion paths. In contrast, Yosida (1955) conducted experiments where the diffusion coefficient was estimated to be 3.5-5 times larger than that for air, while experiments by Sommerfeld et al. (1987) found that the diffusion coefficient was about twice that for air. Yosida (1955) provided a classic description of diffusion being enhanced by water vapor moving between ice grains in a "hand to hand" fashion, thereby shortening the pathway required for water vapor to travel. It is interesting to note that the mechanisms for diffusion argued by Giddings and LaChapelle (1962) and Yosida (1955) both have merit and are competing against one another.

Christon et al. (1994) provided some of the first micromechanics finite element work on mass transfer for snow and generated predictions for the effective diffusion coefficient ranging from 1.0 to 1.93 times the diffusion coefficient for water vapor in air. Christon's results have been criticized, perhaps unfairly in our view, due to the simplistic microstructure models that they were forced to work with at that time. Pinzer et al. (2012) have laid any questions about the influence of microstructure to rest by performing finite element analysis on real snow microstructures generated through Xray computer tomography. Their finite element predictions show a diffusion coefficient for snow to be very nearly that of diffusion of water vapor in air, perhaps an enhancement of 1.05-1.13 for snow compared to diffusion of water vapor in air based on the data provided in Fig. 11 of their work.

Despite wildly more complex microstructures, the results of Pinzer et al. (2012) are largely consistent with the results of Christon et al. (1994). Indeed, in reference to studies on the vapor flux and its dependence on microstructure over time, Pinzer et al. (2012) note that "the flux stays constant in time, despite the dramatic changes in the structure".

The diffusion coefficient given by Eq. (88) divided by the diffusion coefficient of water vapor in air is plotted in Fig. 7 to show Foslien's predicted diffusion enhancement as a function of density at a snow temperature of $263 \mathrm{~K}$. Also plotted in Fig. 7 are the diffusion enhancement ratios of Christon (1990) for two microstructural geometries in their applicable density ranges as well as the more recent predictions of Pinzer et al. (2012) based on real snow microstructures determined from X-ray tomography. Pinzer's results show a range of diffusion enhancement at any given density that represent the bounds of the finite element predictions shown in Fig. 11 of their work.

Foslien's model predicted a maximum diffusion enhancement for snow compared to air of 1.23 with enhancements for typical snow densities in the range from 1.0 to 1.2. These values are in excellent agreement with the numerical predictions of Christon (1990). The favorable comparison is significant in that Christon's simpler microstructures largely resemble a combination of the pore and lamellae structures. The more recent numerical results of Pinzer et al. (2012) fall very much in line with results generated by Christon et al. (1994). In brief, we agree with the view of Pinzer et al. (2012) in that any enhancement of water vapor diffusion in snow compared to diffusion of water vapor in air is minimal. 


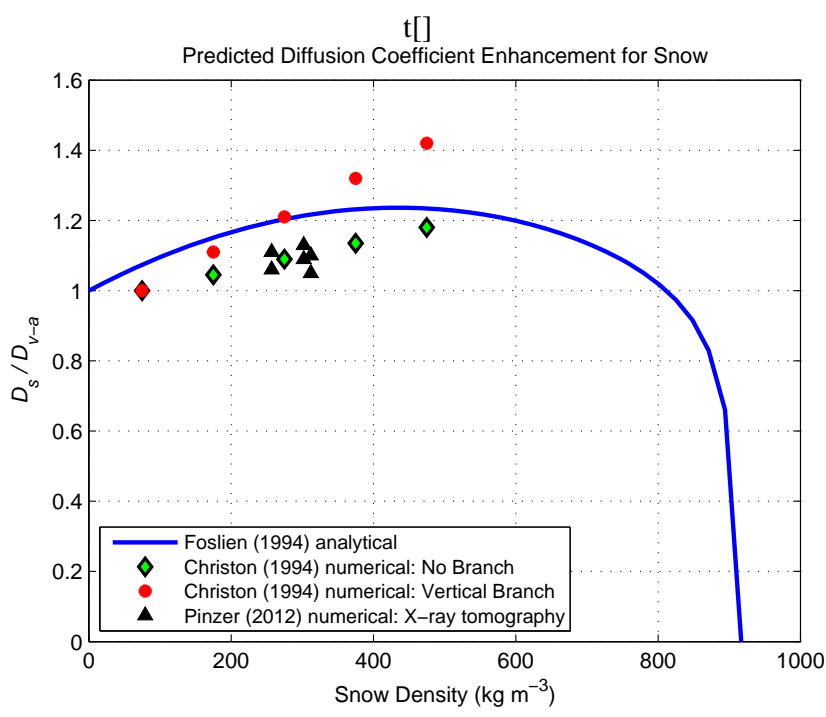

Figure 7. Effective diffusion coefficient enhancement for snow predicted by Foslien (1994) vs. finite element predictions of Christon (1990) and Pinzer et al. (2012).

Calonne et al. (2014a) have recently computed an effective diffusion coefficient for snow by solving the field equations for mass transfer on a series of RVE's computed from 3-D images of snow. The results are interesting in that they show normalized values of $\boldsymbol{D}_{\mathrm{s}}^{\text {eff }}$ starting at 1.0 for humid air alone and steadily decreasing to values as low as 0.2 for snow densities of $500 \mathrm{~kg} \mathrm{~m}^{-3}$.

Calonne et al. (2014a) defined the effective diffusion coefficient of snow as

$\boldsymbol{D}_{\mathrm{s}}^{\mathrm{eff}}=\frac{1}{|V|} \int_{V a} D_{\mathrm{v}-\mathrm{a}}\left(\nabla \boldsymbol{g}_{\mathrm{v}}+\boldsymbol{I}\right) \mathrm{d} V$,

where $\boldsymbol{g}_{\mathrm{v}}$ represents water vapor fluctuation in the air phase.

Following the previous discussion on effective thermal conductivity, the above equation may be written as

$\boldsymbol{D}_{\mathrm{s}}^{\mathrm{eff}}=\phi_{\mathrm{ha}} \frac{1}{\left|V_{\mathrm{a}}\right|} \int_{V a} D_{\mathrm{v}-\mathrm{a}}\left(\nabla \boldsymbol{g}_{\mathrm{v}}+\boldsymbol{I}\right) \mathrm{d} V$.

Introducing the notation

$\boldsymbol{D}_{\mathrm{a}}^{\mathrm{eff}}=\frac{1}{\left|V_{\mathrm{a}}\right|} \int_{V a} D_{\mathrm{v}-\mathrm{a}}\left(\boldsymbol{\nabla} \boldsymbol{g}_{\mathrm{v}}+\boldsymbol{I}\right) \mathrm{d} V$,

as the effective diffusion coefficient of humid air in snow leads to

$\boldsymbol{D}_{\mathrm{s}}^{\mathrm{eff}}=\phi_{\mathrm{ha}} \boldsymbol{D}_{\mathrm{a}}^{\mathrm{eff}}$.

Of interest here is the volume fraction of the humid air constituent leading the volume averaged effective diffusion

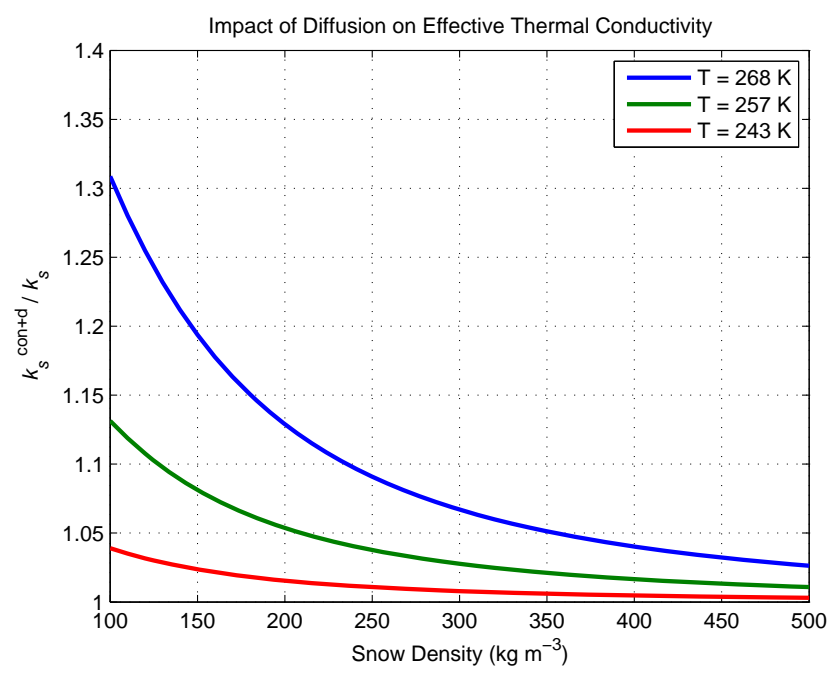

Figure 8. Effective thermal conductivity enhancement due to diffusion predicted by Foslien (1994).

coefficient of the humid air. The linearly decreasing nature of the numerical predictions of Calonne et al. (2014a) as a function $\phi_{\text {ha }}$ suggests $\boldsymbol{D}_{\mathrm{a}}^{\text {eff }}$ is nearly constant for the various samples. Hence, the volume fraction of Eq. (95) acts in a manner similar to the pore microstructure, restricting the pathway for diffusion. While the mechanism to slow diffusion is accounted for by $\phi_{\text {ha }}$ in Eq. (95), it is not readily apparent how this equation accounts for diffusion enhancement via the "hand to hand" mechanism of diffusion described by Yosida (1955), where the pathway of diffusion may be significantly shortened by the ice phase.

We believe an interesting line of inquiry is to compare the finite element results of Pinzer et al. (2012) and Calonne et al. (2014a) for evaluating the effective diffusion coefficient of snow, as their results are fundamentally different. Both use real 3-D snow microstructures and, we believe, a similar finite element numerical approach for the heat and mass transfer solutions. What is the difference then? One possibility is the way in which the diffusion coefficients are computed given a finite element solution. Pinzer et al. (2012) evaluate the mass flux in a slice in the RVE, perpendicular to the temperature gradient - an approach similar to Christon. In contrast Calonne et al. (2014a) utilize Eq. (95) to compute the diffusion coefficient. It would be extremely interesting to explore this topic by taking a single microstructure, evaluating $D_{\mathrm{s}}^{\text {eff }}$ using both approaches.

\subsubsection{Thermal conductivity with diffusion}

The apparent effective thermal conductivity for snow including the enhancement of heat transfer due to mass diffusion is given by Eq. (74). Figure 8 shows the enhancement of thermal conductivity incorporating diffusion effects as a function of density at three different temperatures. For instance, 
at a density of $250 \mathrm{~kg} \mathrm{~m}^{-3}$, the heat transfer enhancement due to diffusion is 9 and $3 \%$ for temperatures of 268 and $257 \mathrm{~K}$, respectively. These values are reasonably consistent with calculated values provided by Riche and Schneebeli (2013) showing latent heat transfer contributions to be approximately 14 and $1 \%$ for temperatures of 268 and $257 \mathrm{~K}$, respectively. Specific densities were not provided for the calculations of Riche and Schneebeli (2013) but the average density of their samples was $254 \mathrm{~kg} \mathrm{~m}^{-3}$.

The analytical predictions of Foslien shown in Fig. 8 suggest the importance of latent heat transfer by diffusion is most prominent in low density snow at temperatures near freezing. In this case, the enhancement of heat transfer due to diffusion may be as high as 30-40\% for low density snow. These results are consistent with the numerical studies of Christon et al. (1994) who note that "the enhancement due to the transport of latent energy is seen to peak at about $40 \%$ of the conduction for the lowest density and the highest base temperature".

In closing, results from the analytical model for the thermal conductivity of snow, $k_{\mathrm{s}}^{\text {eff }}$, and the effective diffusion coefficient for snow, $D_{\mathrm{s}}^{\text {eff }}$, proposed by Foslien are in excellent agreement with cited finite element micromechanics analyses and, further, the parameter predictions are self-consistent with the limiting cases of air and solid ice. The results lend confidence to using the predicted parameters for $k_{\mathrm{s}}^{\text {eff }}$ and $D_{\mathrm{s}}^{\mathrm{eff}}$ over the entire spectrum of temperatures and densities encountered in the macroscale heat and mass transfer analyses presented in Sect. 6.

\section{Numerical results for macroscale heat and mass transfer}

In this section, numerical results of the nonlinear equations (Eqs. 70 and 72) governing heat and mass transfer in a snowpack are presented. The specific problem at hand is to model the heat and mass transfer in a $1 \mathrm{~m}$ deep snow cover with complexities associated with a real snowpack such as dense layers and a time varying surface boundary condition for temperature. A Galerkin finite element method was used to discretize the spatial domain, and the Crank-Nicholson time integration method is used to advance the solution in time. The code used to generate the results of Sect. 6.1 is provided in the Supplement.

Figure 9 shows a schematic of the snow cover characteristics and the associated density profile for the macroscale heat and mass transfer numerical studies. The snowpack had a seasonal snow density of $240 \mathrm{~kg} \mathrm{~m}^{-3}$ with a dense layer starting at $64 \mathrm{~cm}$ and ending at $86 \mathrm{~cm}$. The density was assumed to increase from 240 to $600 \mathrm{~kg} \mathrm{~m}^{-3}$ over $8 \mathrm{~cm}$ with a similar rate of decrease at the top end of the ice crust to a density of $120 \mathrm{~kg} \mathrm{~m}^{-3}$.

The snow density at the ground level was set to solid ice in an effort to impose realistic boundary conditions. If the den-
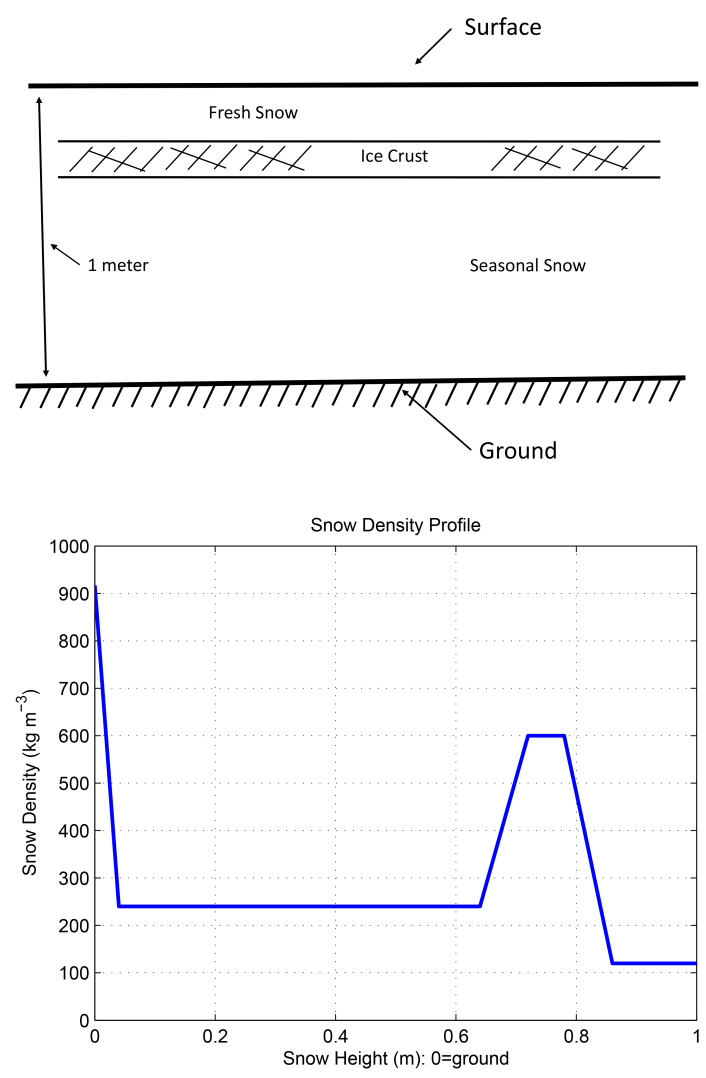

Figure 9. Snow cover characteristics and density profile for the macroscale heat and mass transfer numerical studies.

sity at the ground layer is less than solid ice, the snowpack can be viewed as having no barriers below it. In this case, the model would predict that saturated air enters the snowpack at the ground level with no specified source for this vapor.

Numerical results corresponding to two test cases are presented. The first problem is designed to study the impact of the dense layer on macroscopic temperature gradients and deposition (condensation) and sublimation rates. Colbeck (1993) stressed the importance of developing a theory of snow which incorporates the effect of dense layers on the heat transfer in snow. The second problem focuses on the effect of diurnal temperature fluctuations on the surface of the snowpack. Of interest here are the near surface temperature gradients compared to the average temperature gradient in the snowpack. Condensation and sublimation rates near the surface are also of interest.

\subsection{Influence of an ice crust}

To study the influence of the ice crust on heat and mass transfer, an isothermal snowpack at $0{ }^{\circ} \mathrm{C}$ was initially assumed. Boundary conditions at the ground and surface were 0 and $-20^{\circ} \mathrm{C}$, respectively, thereby initiating a temperature gradient through the snowpack. 


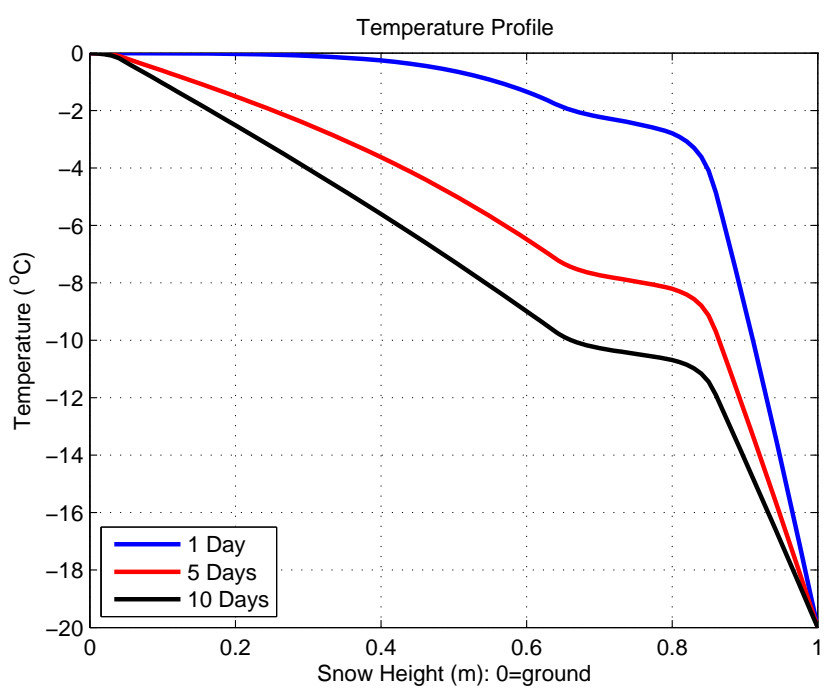

Figure 10. Snowpack temperature profile at 1, 5, and 10 days, respectively.

Figure 10 shows the temperature profile in the snowpack after 1,5 , and 10 days, respectively. In the dense layer of snow, the temperature gradient is noticeably reduced at any time due to the higher thermal conductivity of the dense snow. The dense layer also has the effect of producing a much higher temperature gradient in the near surface fresh snow cover in the pack. Figure 11 shows that, even after 10 days, the temperature gradient near the surface is approximately $60^{\circ} \mathrm{C} \mathrm{m}^{-1}$, three times the average temperature gradient. The high temperature gradients near the surface are even more notable when one considers the significant amplification of temperature gradients that occur at the microscale as discussed in Sect. 2 and demonstrated in Fig. 4 of Pinzer et al. (2012).

Figure 12 shows both condensation and sublimation occurring in the snowpack with significant activity near the dense ice crust. Near the surface, condensation is occurring because water vapor is diffusing toward the surface which is colder and, therefore, an area of lower saturation for the vapor density. The water vapor must then condense if the air is not to become supersaturated. Just below the dense layer, Fig. 12 shows another area of condensation near the dense ice crust. Condensation occurs here due to the decrease in humid air volume fraction in the direction of the water vapor diffusion. If the amount of air available to hold water decreases, then the amount of water vapor must also decrease. Just above the dense layer, the opposite is true. Here, Fig. 12 shows that sublimation is occurring because the vapor volume fraction is increasing in the direction of vapor flow. Sublimation must occur to keep the increasing amount of air saturated.

Local weakening above and below dense layers in a snow cover have been observed (Adams and Brown, 1982). If condensation is known to enhance depth hoar growth (Colbeck, 1983), then the condensation occurring below the dense layer

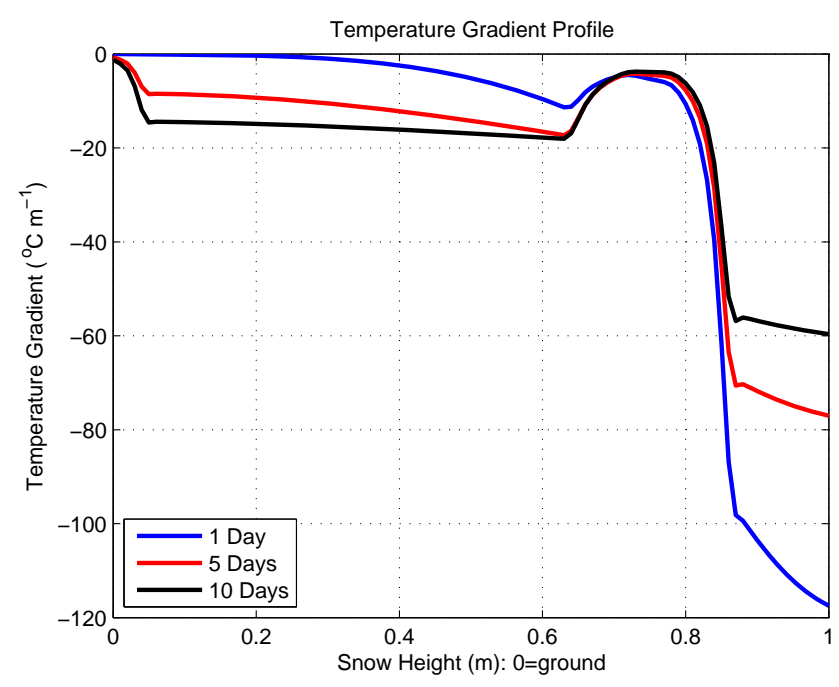

Figure 11. Snowpack temperature gradient profile at 1, 5, and 10 days, respectively.

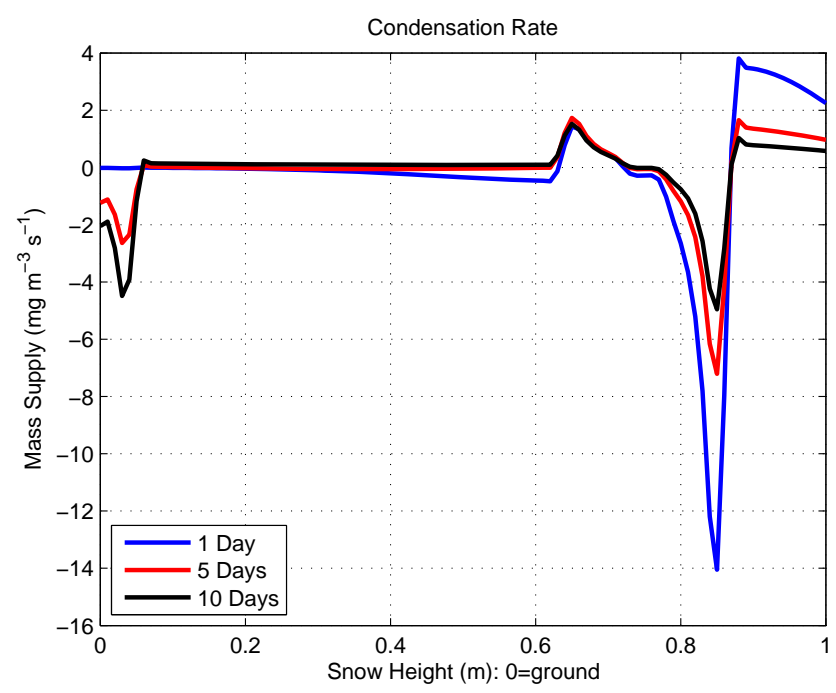

Figure 12. Snowpack condensation rates at 1,5 , and 10 days, respectively. Positive values indicate condensation while negative values indicate sublimation.

in Fig. 12 could contribute to the weakening observed in this region of a snowpack. Temperature gradients are relatively low below the ice crust, although in the range of where faceted crystal growth has been observed in field studies. The sublimation occurring above the dense layer may contribute to any snowpack weakness observed here.

Sublimation is also observed near the ground after 10 days and appears to be increasing with time. At a sublimation rate of $4 \mathrm{mg} \mathrm{m}^{-3} \mathrm{~s}^{-1}$ occurring just above the ground after 10 days, a decrease in density of $1 \mathrm{~kg} \mathrm{~m}^{-3}$ would take about 3 days. This slow rate of change in density is consistent with experimental studies on temperature gradient metamorphism, where little change in density is observed despite 


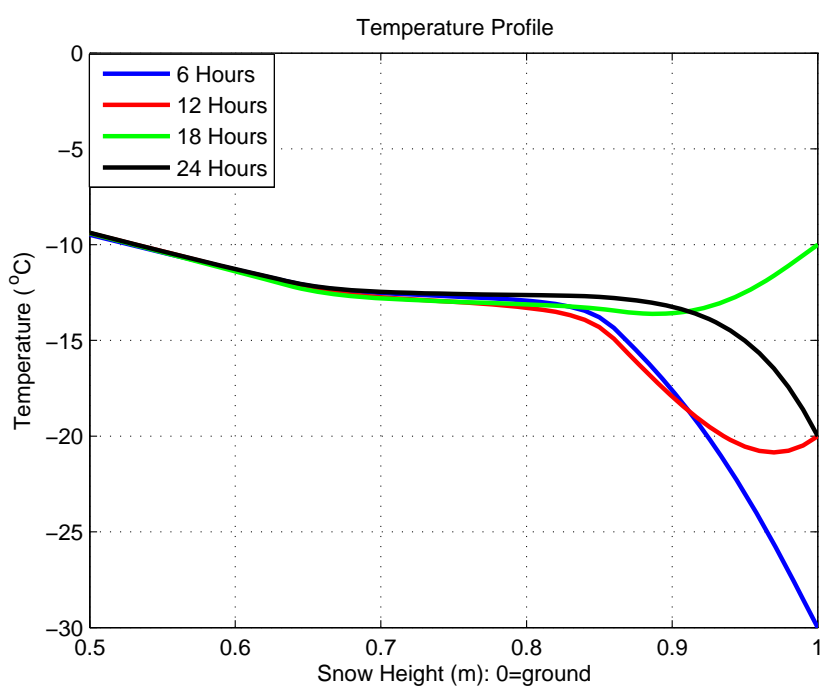

Figure 13. Snowpack temperatures over a $24 \mathrm{~h}$ period with time dependent surface boundary conditions given by Eq. (98).

the dramatic changes in the crystal structure of snow (Pinzer et al., 2012).

\subsection{Effect of diurnal temperatures}

To study diurnal temperature effects, the surface of the snowpack was subjected to a harmonic temperature boundary condition given by

$\theta(1, t)=-20-10 \sin \frac{\pi t}{43200}$,

with the lower surface temperature held fixed at $0{ }^{\circ} \mathrm{C}$. The boundary condition given by Eq. (98) has a mean value of $-20^{\circ} \mathrm{C}$ with a $10^{\circ} \mathrm{C}$ fluctuation about the mean. Initial conditions for the temperature through the snowpack were specified based on the average temperature gradient of $-20{ }^{\circ} \mathrm{C} \mathrm{m}^{-1}$ at time $t=0$.

Figure 13 shows the temperature profile over a $24 \mathrm{~h}$ period at day 5, after any transients have disappeared. Only the upper half meter of the snowpack is shown. Strong temperature gradients are observed in the fresh snow near the surface, with gradients as high as $-150{ }^{\circ} \mathrm{C} \mathrm{m}^{-1}$ as shown in Fig. 14 . The large temperature gradients found near the surface are also accompanied by active condensation and sublimation throughout a $24 \mathrm{~h}$ cycle (Fig. 15). Both the temperature gradient and the mass exchange are likely to impact microstructural changes that occur in the near surface snow cover.

\section{Summary}

We have developed a macroscale mixture theory analysis for modeling condensation and sublimation rates in a snow cover under temperature gradient conditions. The theory is general enough to accommodate arbitrary density profiles with any

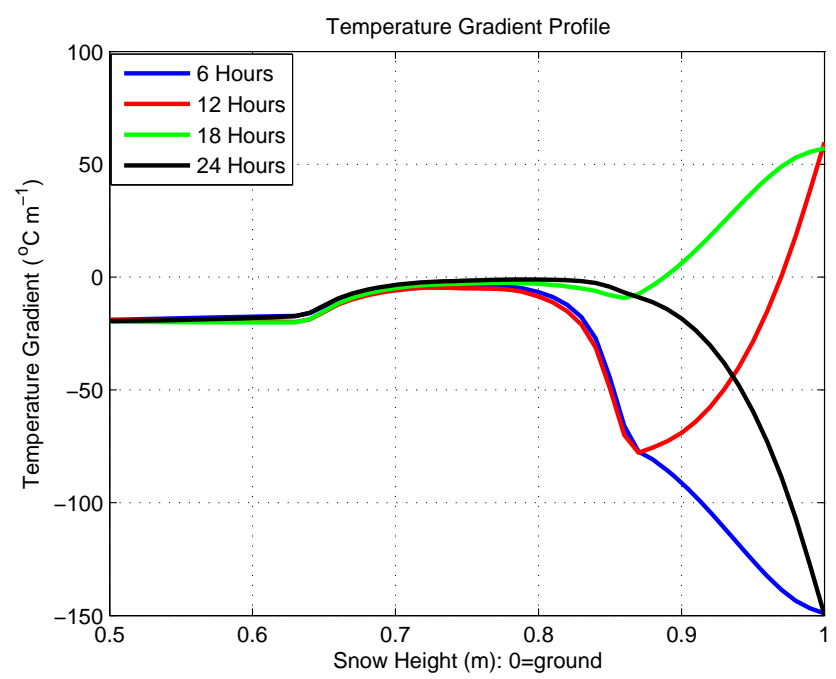

Figure 14. Snowpack temperature gradients over a $24 \mathrm{~h}$ period with time dependent surface boundary conditions given by Eq. (98).

desired time dependent surface boundary conditions. Condensation and/or sublimation is observed near the ground and the surface as well as immediately above and below dense layers such as ice crusts. Macroscale temperature gradients can be surprisingly high compared to the average temperature gradient in the snowpack.

A quantitatively correct macroscale analysis of constituent mass exchange brings out an interesting microscale analysis problem that is worthy of attention. Recent numerical studies of heat and mass transfer at the microscale begin by solving the steady state diffusion equation for heat transfer with the appropriate thermal properties of ice and humid air. Dirichlet boundary conditions for temperature are specified at the top and bottom of the RVE to simulate a desired temperature gradient. Once the local temperature field is determined, a solution for mass transport is obtained for the humid air phase. The vapor density boundary conditions are specified at the ice/air interface by assuming the vapor density is saturated, thereby a function of temperature only. Therefore, the mass flux in such an analysis is essentially dictated by the macroscale temperature gradient.

The mixture theory results for macroscale deposition and or sublimation tell an entirely different story. For example, consider the effects of diurnal temperatures on heat and mass transfer near the surface as seen in Figs. 14 and 15. Figure 14 shows strong negative temperature gradients at 6 and $24 \mathrm{~h}$ between $x=0.9 \mathrm{~m}$ and $x=1.0 \mathrm{~m}$ (the region bounded by the ice crust and the surface). Now, examine Fig. 15 showing mass exchange at the same time periods and snow depth. The $6 \mathrm{~h}$ plot shows deposition is occurring while the $24 \mathrm{~h}$ plot shows sublimation is occurring. The boundary conditions used for microscale analyses of an RVE described above cannot capture this interesting phenomenon as the results are driven by temperature gradient only. 


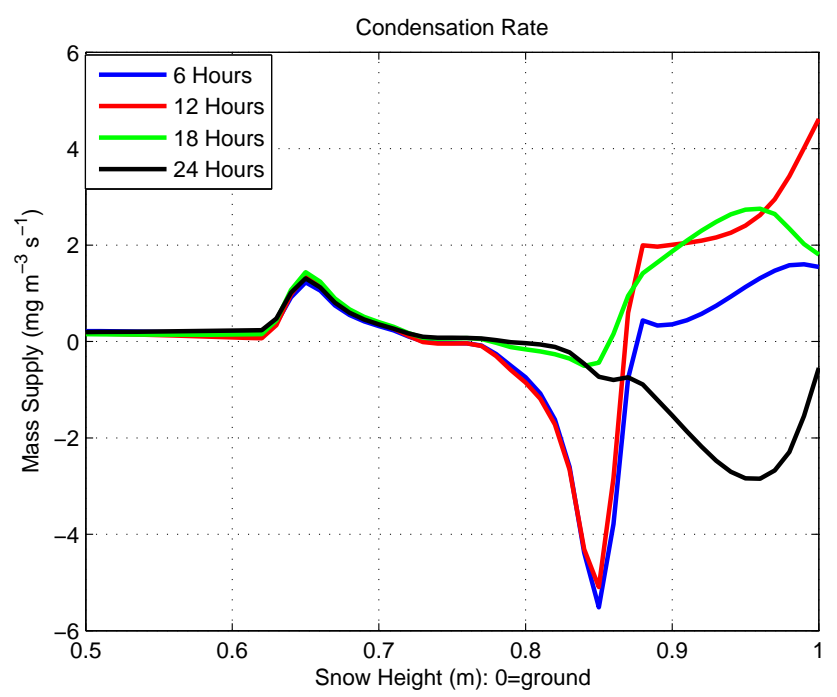

Figure 15. Snowpack condensation rates over a $24 \mathrm{~h}$ period based on the diurnal boundary condition of Eq. (98). Positive values indicate condensation while negative values indicate sublimation.

The divergent behavior of macroscale deposition/sublimation phenomena from the macroscale temperature gradient should come as no surprise from the mixture theory analysis. Indeed, the ability to show this phenomenon is the fundamental driver for developing a mixture theory to begin with. If this were not the case, one could simply generate a thermal profile from the energy equation and move on.
Given the mixture theory results described above, it would be interesting to investigate a transient microscale (RVE) solution exploring the effects of imposing mass flux boundary conditions for the vapor while using the saturated vapor density (based on the temperature field) as an initial condition for the humid air. Perhaps such an analysis could help explain microstructural evolution (kinetic growth vs. sintering) under conditions where specifying the temperature gradient alone is unable to do so. Christon (1990) developed a fully coupled heat and mass transfer formulation, although boundary conditions involved temperature and temperature gradient only.

Knowledge of the condensation or sublimation rates at the macroscale provides the foundation for a fully coupled solution of heat and mass transfer at the microscale. In this spirit, the mixture theory for snow can simulate interesting macroscale problems featuring terrain changes, ice crusts, surface effects, snow storm deposition, extreme temperatures, etc. Heat and mass transfer results from a mixture theory analysis could then be used as inputs for a host of interesting microstructural studies.

Finally, analytical expressions of thermal conductivity and the effective diffusion coefficient for snow were motivated from simple microstructures whose heat and mass transfer are grounded in first principles. The equations developed provide an elegant path to modeling thermal conductivity and the effective diffusion coefficient of snow that are needed for the macroscale mixture theory analysis. 


\section{Appendix A}

Table A1. Nomenclature.

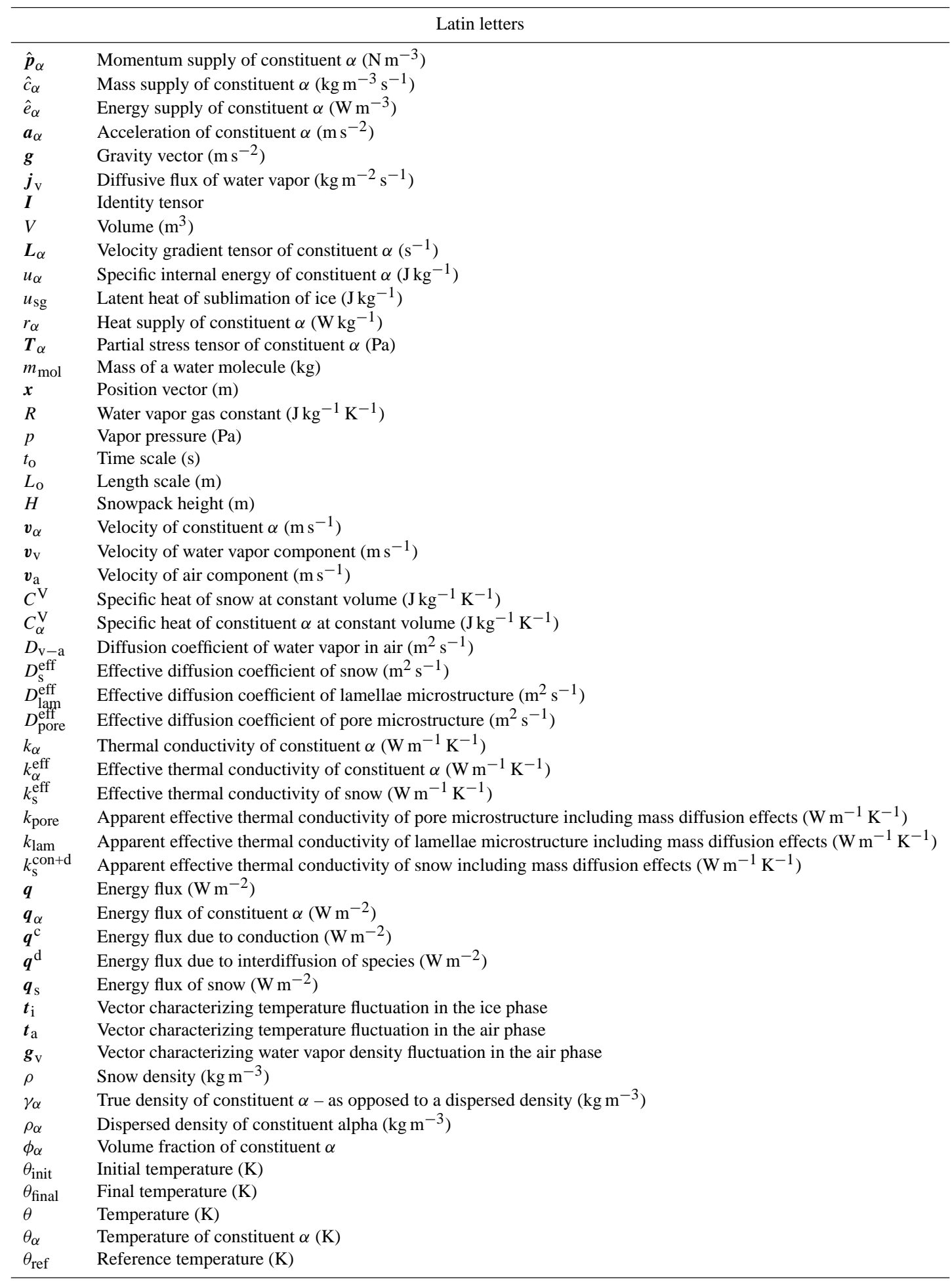


Table A1. Continued.

\begin{tabular}{|c|c|}
\hline \multicolumn{2}{|r|}{ Greek symbols } \\
\hline$\chi_{\mathrm{i}}$ & Indicator function for the ice constituent \\
\hline$\alpha_{\mathrm{c}}$ & Condensation coefficient \\
\hline$\Omega$ & Boltzman's constant $\left(\mathrm{J} \mathrm{K}^{-1}\right.$ molecule $\left.^{-1}\right)$ \\
\hline$\xi$ & Specific surface area of snow $\left(\mathrm{m}^{-1}\right)$ \\
\hline \multicolumn{2}{|r|}{ Subscripts } \\
\hline$\alpha$ & Arbitrary constituent \\
\hline a & Air component \\
\hline ha & Humid air constituent \\
\hline i & Ice constituent \\
\hline $\mathrm{s}$ & Snow \\
\hline $\mathrm{v}$ & Water vapor component \\
\hline $\mathrm{m}$ & Indicates variable applies at the microscale \\
\hline ref & Reference \\
\hline \multicolumn{2}{|r|}{ Superscripts } \\
\hline eff & Effective - applies at the macroscale \\
\hline sat & Saturated water vapor in air \\
\hline$*$ & Dimensionless variable \\
\hline \multicolumn{2}{|r|}{ Operators } \\
\hline$\nabla$ & Gradient operator \\
\hline$\nabla_{x}$ & Gradient operator applied at the microscale \\
\hline
\end{tabular}




\section{The Supplement related to this article is available online at doi:10.5194/tc-9-1857-2015-supplement.}

Edited by: M. Schneebeli

\section{References}

Adams, E. and Brown, R.: Further results on studies of temperature gradient metamorphism, J. Glaciol., 28, 205-209, 1982.

Adams, E. and Brown, R.: A mixture theory for evaluating heat and mass transport processes in nonhomogeneous snow, Continuum Mech. Therm., 2, 31-63, 1990.

Albert, M. R. and McGilvary, W. R.: Thermal effects due to air flow and vapor transport in dry snowl, J. Glaciol., 38, 273-281, 1992.

Auriault, J.-L., Boution, C., and Geindreau, C.: Homogenization of Coupled Phenomena in Heterogeneous Media, John Wiley and Sons, London, 2009.

Bader, H. P. and Weilenmann, P.: Modeling temperature distribution, energy and mass flow in a (phase-changing) snowpack, I. Model and case studies, Cold Reg. Sci. Technol., 20, 157-181, doi:10.1016/0165-232X(92)90015-M, 1992.

Bird, R. B., Stewart, W. E., and Lightfoot, E.: Transport Phenomena, John Wiley and Sons, New York, 1960.

Calonne, N., Flin, F., Morin, S., Lesaffre, B., Roscoat, R., and Geindreau, C.: Numerical and experimental investigations of the effective thermal conductivity of snow, Geophys. Res. Lett., 38, L23501, doi:10.1029/2011GL049234, 2011.

Calonne, N., Geindreau, C., and Flin, F.: Macroscopic modeling for heat and water vapor transport in dry snow by homogenization, J. Phys. Chem. B, 118, 13393-13403, doi:10.1021/jp5052535, 2014a.

Calonne, N., Flin, F., Geindreau, C., Lesaffre, B., and Rolland du Roscoat, S.: Study of a temperatue gradient metamorphism of snow from 3-D images: time evolution of microstructures, physical properties and their associated anisotrpy, The Cryosphere, 8 , 2255-2274, doi:10.5194/tc-8-2255-2014, 2014b.

Christon, M.: 3-D transient microanalysis of multi-phase heat and mass transfer in ice lattices, $\mathrm{PhD}$ thesis, Colorado State University, Fort Collins, CO, USA, 1990.

Christon, M., Burns, P., and Sommerfeld, R.: Quasi-steady temperature gradient metamorphism in idealized, dry snow, Numer. Heat Tr. A-Appl., 25, 259-278, doi:10.1080/10407789408955948, 1994.

Colbeck, S.: Theory of metamorphism of dry snow, J. Geophys. Res., 88, 5475-5482, 1983.

Colbeck, S.: Snow-crystal growth with varying surface temeperatures and radiation penetration, J. Glaciol., 35, 23-29, 1989.

Colbeck, S.: The vapor diffusion coefficient for snow, Water Resour. Res., 29, 109-115, 1993.

Delaney, L. J., Houston, R. W., and Eagleton, L. C.: The rate of vaporization of water and ice, Chem. Eng. Sci., 19, 105-114, doi:10.1016/0009-2509(64)85115-0, 1964.

de Quervain, M.: On the metomorphosis of snow, Water Resour. Res., 29, 109-115, 1963.

Dorsey, N.: Properties of Ordinary Water-Substance, Hafner, New York, 1968.

Flin, F. and Brzoska, J.: The temperature-gradient metamorphism of snow: vapour diffusion model and application to tomographic images, Ann. Glaciol., 49, 17-21, 2008.
Foslien, W.: A modern mixture theory applied to heat and mass transfer in snow, MS thesis, University of Wyoming, Laramie, WY, USA, 1994.

Giddings, J. and LaChapelle, W.: The formation rate of depth hoar, J. Geophys. Res., 67, 2377-2383, 1962.

Gray, J. M. and Morland L. W.: A dry snowpack model, Cold Reg. Sci. Technol., 22, 135-148, doi:10.1016/0165-232X(94)900256, 1994.

Hansen, A.: Reexamining some basic definitions of modern mixture theory, Int. J. Eng. Sci., 27, 1531-1544, doi:10.1016/00207225(89)90146-8, 1989.

Hansen, A., Damson, M., Donovan, R., Horning, D., and Walker, J.: Some notes on a volume fraction mixture theory and a comparison with the kinetice theory of gases, Int. J. Eng. Sci., 29, 561573, doi:10.1016/0020-7225(91)90061-7, 1991.

Hobbs, P.: Ice Physics, Clarendon Press, Oxford, 1974.

Löwe, H. R., Riche, F., and Schneebeli, M.: A general treatment of snow microstructure exemplified by an improved relation for thermal conductivity, The Cryosphere, 7, 1473-1480, doi:10.5194/tc-7-1473-2013, 2013.

McComb, T., Rimmer, A., Rodgers, M., Turver, K., and Vickers, A.: A mathematical model for the prediction of temperature in a dry snow layer, Cold Reg. Sci. Technol., 20, 247-259, 1992.

Morland, L. W., Kelly, R. J., and Morris, E. M.: A mixture theory for a phase changing snowpack, Cold Reg. Sci. Technol., 17, 271285, doi:10.1016/S0165-232X(05)80006-0, 1990.

Özdemir, W., Brekelmans, A., and Geers, M. G. D.: Computational homogenization for heat conduction in heterogeneous solids, Int. J. Numer. Meth. Eng., 73, 185-204, 2008.

Pinzer, B. and Schneebeli, M.: Snow metamorphism under alternating temperature gradients: morphology and recrystalization in surface snow, Geophys. Res. Lett., 36, L23503, doi:10.1029/2009GL039618, 2009.

Pinzer, B. R., Schneebeli, M., and Kaempfer, T. U.: Vapor flux and recrystallization during dry snow metamorphism under a steady temperature gradient as observed by time-lapse microtomography, The Cryosphere, 6, 1141-1155, doi:10.5194/tc-61141-2012, 2012.

Riche, F. and Schneebeli, M.: Thermal conductivity of snow measured by three independent methods and anisotropy considerations, The Cryosphere, 7, 217-227, doi:10.5194/tc-7-217-2013, 2013.

Schertzer, R. and Adams, E.: Anisotropic thermal conductivity model for dry snow, Cold Reg. Sci. Technol., 69, 122-128, 2011.

Sommerfeld, R., Friedman, I., and Nilles, M.: The fractionation of natural isotopes during temperature gradient metamorphism of snow, in: The Fractionation of Natural Isotopes During Temperature Gradient Metamorphism of Snow, D. Reidel Publishing, Boston, 95-105, 1987.

Underwood, E.: Quantitative Stereology, Addison-Wesley, Reading, Mass., USA, 1970.

Yosida, Z.: Physical studies of deposited snow: thermal properties I, Tech. rep., Institute of low temperature science, Hokkaido University, Japan, 1955. 